\title{
Adaptive Control Design and Evaluation for Multibody High-speed Train Dynamic Models
}

\author{
Zehui Mao, Gang Tao, Fellow, IEEE, Bin Jiang, Fellow, IEEE, Xing-Gang Yan
}

\begin{abstract}
In this paper, the adaptive tracking control problem is investigated for multibody high-speed train dynamic model in the presence of unknown parameters, which is an open adaptive control problem. A 4-car train unit model with input signals acting on the 2nd and 3rd cars and output signals being the speeds of the 1st and 3rd cars is chosen as a benchmark model, in which the aerodynamic resistance force is also considered. To handel the nonlinear term, the feedback linearization method is employed to decompose the system into a control dynamics subsystem and a zero dynamics subsystem. A new and detailed stability analysis is conducted to show that such a zero dynamic system is Lyapunov stable and is also partially input-to-state stable under the condition that the speed error between the 1st and 3rd cars is exponentially convergent (to be ensured by a nominal controller) or belongs to the $L^{1}$ signal space (to be achieved by a properly designed adaptive controller). The system configuration leads to a relative degree 1 subsystem and a relative degree 2 subsystem, for which different feedback linearization-based adaptive controllers and their nominal versions are developed to ensure the needed stabilization condition, the desired closed-loop system signal boundedness and asymptotic output speed tracking. Detailed closed-loop system stability and tracking performance analysis are given for the new control schemes. Simulation results from a realistic train dynamic model are presented to verify the desired adaptive control system performance.
\end{abstract}

Index Terms-Adaptive control, output tracking, high-speed train, multibody models, feedback linearization, zero dynamics

\section{INTRODUCTION}

With the developments of railway systems, high-speed trains are being updated to run at higher speeds and more loading capacities. Speed tracking is the fundamental requirement for the punctuality of the operation of a train, which leads to the increasing of the automatic train operation control capabilities of high-speed trains. Great efforts have been devoted to the control design for high-speed trains, such as active communication-based control [1], data-based control [2], iterative learning control [3], robust control [4]- [5], adaptive control [6], and so on.

In studies of train control, there are mainly two types of models used in the literatures, namely, the single mass point

This work was supported in part by the National Natural Science Foundation of China under Grant 61922042 and by a Qing Lan Project, in part by the USA NSF under Grant ECCS1509704 (for the second author), and in part by the Fundamental Research Funds for the Central Universities under Grant NC2020002, NP2020103. (Corresponding author: Gang Tao.)

Z. Mao and B. Jiang are with College of Automation Engineering, Nanjing University of Aeronautics and Astronautics, Nanjing, 210016, China, Email: zehuimao@nuaa.edu.cn (Z. Mao), binjiang@nuaa.edu.cn (B. Jiang).

G. Tao is with Department of Electrical and Computer Engineering, University of Virginia, Charlottesville, VA 22904, U.S.A, Email: gt9s@ virginia.edu.

X. Yan is with School of Engineering and Digital Arts, University of Kent, Canterbury, Kent CT2 7NT, United Kingdom, Email: x.yan@kent.ac.uk. model and the cascade mass point model [7]. The former considers the whole train as one single mass point and ignores in-train dynamics of the train, see [8]- [10]. The latter models a train as individual mass points that are inter-connected via springlike couplers, see [11]- [13]. For the traditional trains, such as heavy-haul-trains, the traction force only acts on the head car, i.e., only the head car is the power car, so that the connections between each cars should be ensured to tolerant the traction force and do not break under the train operating. The single mass point model is enough to study the control problem.

Nowadays, to achieve the high speed for trains, the powers are distributed in a train, i.e., for a high-speed train, several cars are power cars and others are trailer cars, which makes the inter-force generated by the connections cannot be ignored in control design. This results in the cascade mass point model for controller design, for which the multibody model (i.e., cascade mass point model) is wildly employed [15]- [17]. In [18], a distributed and fault-tolerant control design approach is presented to develop tracking and braking control schemes for high-speed trains with a multibody system. In [19], a tracking controller is designed for the high-speed trains using the multiagent technology. In [20], a robust control criterion for the velocity tracking control of high-speed trains is developed based on a multi-particle model. Among these results, it is assumed that the considered models are always full-actuated with the traction forces acting on every car and being designed separately, or the condition that the train is operating under the equilibrium state. Therefore, the in-train dynamics related to the inter-forces have not been fully analyzed.

In this paper, a 4-car train unit from $\mathrm{CRH}_{2}$ (China Railway High-speed 2), with the 2nd and 3rd cars being power cars, is chosen as the benchmark model, see Fig. 1, which is an underactuated 4-car system containing the in-train dynamics. These in-train dynamics are the "internal" behaviors that cannot be controlled by the input signals directly, which are called zero dynamics [21]- [22]. The stability performance for the zero dynamics is a key part to ensure the effectiveness of the controller. However, this analysis for the underactuated 4-car train model has not been obtained in the current results. Moreover, considering the fact that actual values of system parameters are not obtained easily, and the trajectory tracking problem is an important topic for train control systems, the adaptive control technique is an effect way to deal with the unknown parameters to achieve high tracking performance [23]- [27]. Thus, the adaptive tracking control for an underactuated 4-car system with unknown parameters is of both theoretical challenges and practical importance. 


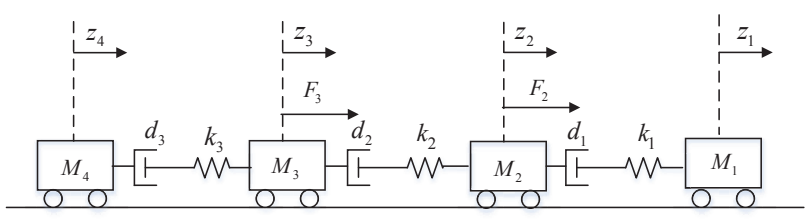

Fig. 1: A 4-car rail vehicle

The purpose of this paper is to solve an open adaptive control problem for high-speed trains represented by a nonlinear underactuated 4-car system with unknown system parameters to achieve the speed tracking. The main contributions of this paper are as follows:

(i) A typical 4-car train unit from a real $\mathrm{CRH}_{2}$ high-speed train is chosen as a benchmark model, which is modelled as a nonlinear underactuated 4-car system with unknown system parameters. The feedback linearization method is employed to decompose the system into a control dynamics subsystem and a zero dynamics subsystem.

(ii) A new and detailed stability study of the zero dynamics subsystem is presented, while a new partial input-to-state stability property is given and the stabilization condition is derived. Moreover, the physical meaning of such partial input-to-state stability is clarified.

(iii) For the relative degrees 1 and 2 subsystems from the system configuration, the new feedback linearizationbased adaptive controllers with the key stability properties are clarified and achieved to guarantee the stability of the zero dynamics subsystem and the tracking performance, respectively. Also, the stability properties of the corresponding nominal controller are presented.

The rest of the paper is organized as follows: Section II describes the 4-car nonlinear benchmark model and formulates the adaptive tracking control problem. Section III decomposes the system into a control dynamics subsystem and a zero dynamics subsystem by feedback linearization. Section IV studies the stability analysis for the zero dynamics subsystem. Sections V provides the adaptive tracking control scheme. Section VI includes the simulation study, followed by conclusions in Section VII.

\section{System Description AND PROBlem Formulation}

A high-speed train consists of several same type units, in which the typical unit, shown in Fig. 1 is made up of 4 cars with the 2 nd and 3 rd ones being power cars. Here, this kind of unit is chosen as the benchmark model to study the tracking control problem.

\section{A. Individual Car Model of Motion}

To obtain the train dynamic model, it is necessary to analyze the forces acting on the cars. A high-speed train consisting of $n$ cars can be considered as $n$ mass points connected by $n-1$ couplers. For the $i$-th car, the longitudinal dynamic movement can be described by [28]- [29]:

$$
M_{i} \ddot{z}_{i}(t)=F_{i}(t)+F_{i n_{i-1}}(t)-F_{i n_{i}}(t)-F_{r_{i}}(t),
$$

where $z_{i}$ is the position of the $i$-th car, $M_{i}$ is the mass of the $i$-th car, $F_{i}$ is the traction force, which is only for the power car, $F_{i n_{i}}$ is the in-train force between the $i$-th and $(i+1)$ th cars, $F_{r_{i}}$ is the general resistance. The resistance $F_{r_{1}}$ is different from the resistances of the other cars.

The general resistance $F_{r_{i}}(t)$ is approximated by a function (see [18], [20], [30] and [31]):

$$
F_{r_{i}}(t)= \begin{cases}a_{r_{i}}+b_{r_{i}} v_{i}(t)+c_{r_{i}} v_{i}^{2}(t), & i=1, \\ a_{r_{i}}+b_{r_{i}} v_{i}(t), & i=2, \ldots, n,\end{cases}
$$

where $v_{i}(t)$ is the speed of the $i$-th car, $a_{r_{i}}, b_{r_{i}}$ and $c_{r_{i}}$ are the resistance coefficients. The term $c_{r_{i}} v_{i}^{2}(t)$ represents aerodynamic drag, while the remaining terms $a_{r_{i}}$ and $b_{r_{i}} v_{i}(t)$ are considered to be rolling mechanical resistances.

The in-train forces are generated through the couplers between cars, which can be modelled by [30]

$$
F_{i n_{i}}(t)=k_{i}\left(z_{i}-z_{i+1}\right)+d_{i}\left(\dot{z}_{i}-\dot{z}_{i+1}\right),
$$

where $k_{i}$ and $d_{i}$ are the spring and damping constants, $\dot{z}_{i}, \dot{z}_{i+1}$ and $z_{i}, z_{i+1}$ are the speeds and the positions of the $i$-th and $(i+1)$-th cars, respectively.

According to the well-developed measurement techniques for high-speed trains, such as the leaky coaxial synthesized optical cable and the fibre optic gyroscope inertial navigation system [29]- [31], the position $z_{i}$ and speed $\dot{z}_{i}$ of each car can be measured accurately [5].

\section{B. Train Model of Motion}

Based on the force analysis, the motion dynamic equations of the train with $n$ cars are given by

$$
\begin{aligned}
M_{1} \ddot{z}_{1}(t)= & F_{1}(t)-k_{1}\left(z_{1}(t)-z_{2}(t)\right)-d_{1}\left(\dot{z}_{1}(t)-\dot{z}_{2}(t)\right) \\
& -\left(a_{r_{1}}+b_{r_{1}} \dot{z}_{1}(t)+c_{r_{1}} \dot{z}_{1}^{2}(t)\right) \\
M_{i} \ddot{z}_{i}(t)= & F_{i}(t)-k_{i}\left(z_{i}(t)-z_{i+1}(t)\right)-k_{i-1}\left(z_{i}(t)-z_{i-1}(t)\right) \\
& -d_{i}\left(\dot{z}_{i}(t)-\dot{z}_{i+1}(t)\right)-d_{i-1}\left(\dot{z}_{i}(t)-\dot{z}_{i-1}(t)\right) \\
& -\left(a_{r_{i}}+b_{r_{i}} \dot{z}_{i}(t)\right), \quad i=2, \ldots, n-1 \\
M_{n} \ddot{z}_{n}(t)= & F_{n}(t)-k_{n-1}\left(z_{n}(t)-z_{n-1}(t)\right) \\
& -d_{n-1}\left(\dot{z}_{n}(t)-\dot{z}_{n-1}(t)\right)-\left(a_{r_{n}}+b_{r_{n}} \dot{z}_{n}(t)\right)
\end{aligned}
$$

where $\dot{z}_{i}$ and $z_{i}$ are the speed and the position of the $i$-th car, respectively, $k_{i}$ and $d_{i}$ are the spring and damping constants of the coupler systems, $M_{i}$ and $F_{i}$ are the mass and traction force of the $i$-th car, respectively.

\section{Benchmark System}

Here, the 4-car train model shown in Fig. 1 is chosen as the benchmark system to study the adaptive control problem. From (4)-(6), it can be seen when the speeds are non-zero, the positions of cars may be infinity, which means the states $z_{1}(t), z_{2}(t), z_{3}(t)$ and $z_{4}(t)$ go to infinity, if $\dot{z}_{1}(t), \dot{z}_{2}(t)$, $\dot{z}_{3}(t)$ and $\dot{z}_{4}(t)$ do not go to zero. It is impossible to analyze and obtain the stability property for the states $z_{1}(t), z_{2}(t)$, $z_{3}(t)$ and $z_{4}(t)$ in the train dynamic model (4)-(6). However, the relative positions, such as, $z_{1}(t)-z_{2}(t), z_{2}(t)-z_{3}(t)$ and $z_{3}(t)-z_{4}(t)$, between each cars should and must be bounded. Otherwise the connections between adjoining cars 
will be broken. Thus, the relative positions are chosen as states to model the train dynamics for an adaptive controller design.

For the 4-car benchmark model, choose the system state $x(t)=\left[x_{1}(t), \ldots, x_{8}(t)\right]^{T}=\left[z_{1}(t), \quad \dot{z}_{1}(t), \quad z_{1}(t)-\right.$ $\left.z_{2}(t), \quad \dot{z}_{2}(t), \quad z_{2}(t)-z_{3}(t), \quad \dot{z}_{3}(t), \quad z_{3}(t)-z_{4}(t), \quad \dot{z}_{4}(t)\right]^{T} \in$ $R^{8}$, and set $m_{p}=\frac{1}{M_{p}}, d_{p q}=\frac{d_{p}}{M_{q}}, k_{p q}=\frac{k_{p}}{M_{q}}, a_{p}=\frac{a_{r_{p}}}{M_{p}}$, $b_{p}=\frac{b_{r_{p}}}{M_{p}}$, and $c_{1}=\frac{c_{r_{1}}}{M_{1}}$, with $p, q=1,2,3,4$. From (4)-(6), the dynamic equation for 4-car train unit in Fig. 1 can be written as

$$
\dot{x}(t)=A x(t)+B F(t)-B_{a}-B_{c} x_{2}^{2}(t),
$$

where $A$ is given in (8),

$$
\begin{aligned}
B & =\left[\begin{array}{cccccccc}
0 & 0 & 0 & m_{2} & 0 & 0 & 0 & 0 \\
0 & 0 & 0 & 0 & 0 & m_{3} & 0 & 0
\end{array}\right]^{T}, \\
F(t) & =\left[\begin{array}{c}
F_{2}(t) \\
F_{3}(t)
\end{array}\right], \\
B_{a} & =\left[\begin{array}{llllllll}
0 & a_{1} & 0 & a_{2} & 0 & a_{3} & 0 & a_{4}
\end{array}\right]^{T}, \\
B_{c} & =\left[\begin{array}{llllllll}
0 & c_{1} & 0 & 0 & 0 & 0 & 0 & 0
\end{array}\right]^{T},
\end{aligned}
$$

with $d_{p q}>0, k_{p q}>0, m_{p}>0, a_{p}, b_{p}$ and $c_{1}$ being unknown parameters.

Remark 1: There are several basic units of high-speed trains, such as a unit containing 4 cars with the 1st, 2nd and 3rd cars being power cars, or the 3rd and 4th cars being power cars, or a unit containing 3 cars with the 1 st and 3 rd cars being power cars, and so on. In this paper, we mainly develop an adaptive control framework for the high-speed train benchmark model (7), which can be easily extended to the other highspeed train models.

\section{Control objective}

In practice, the speed and position of each car can be available, that is, the states $x(t)$ of the system (7) are all measurable, among which any state can be chosen as the controlled variable for adaptive controller design. As the trajectory tracking is the important task for trains, and if the head car (1st car) can track the desired speed trajectory and the connections work normally, the whole train could achieve the tracking performance. Moreover, there is a nonlinear term $c_{1} x_{2}^{2}(t)$ in the 1 st car, while the inputs $F_{2}(t)$ and $F_{3}(t)$ do not act on the 1st car directly. From the practical application, in order to deal with the nonlinear term $c_{1} x_{2}^{2}(t)$ and the unknown system parameters, we choose the speeds of the 1 st and $3 \mathrm{rd}$ cars as the controlled variables, which can be considered as the output of the system (7), i.e.,

$$
y(t)=\left[\begin{array}{c}
y_{1}(t) \\
y_{2}(t)
\end{array}\right]=\left[\begin{array}{c}
x_{2}(t) \\
x_{6}(t)
\end{array}\right]=\left[\begin{array}{c}
\dot{z}_{1}(t) \\
\dot{z}_{3}(t)
\end{array}\right] .
$$

Therefore, the control objective of this paper can be summarized as follows: an adaptive controller is to be designed for the high-speed train system (7) to make the states (outputs) $\dot{z}_{1}(t)$ and $\dot{z}_{3}(t)$ tracking the same desired speed signal $v_{m}(t)$, and simultaneously to keep the states $z_{1}(t)-z_{2}(t), \dot{z}_{2}(t)$, $z_{2}(t)-z_{3}(t), z_{3}(t)-z_{4}(t)$, and $\dot{z}_{4}(t)$ bounded, in the presence of the nonlinear term $c_{1} x_{2}^{2}(t)$ and unknown system parameters $d_{p q}, k_{p q}, m_{p}$ and $a_{p}$ for $p, q=1,2,3,4$.

\section{SySTEM FEEDBACK LINEARIZATION}

It can be seen that the high-speed train dynamic system (7) is a nonlinear system with the nonlinear term $c_{1} x_{2}^{2}(t)$ in the dynamics equation $\dot{x}_{2}(t)$. To handle this class of nonlinear term, the feedback linearization method will be employed to decompose this system (7) into a control dynamics subsystem and a zero dynamics subsystem.

\section{A. Relative Degrees}

As described in [21], the vector relative degree is crucial to feedback linearization-based adaptive controller design for nonlinear systems, which will be calculated for the system (7) to achieve the linearization.

From (7) and (11), the train nonlinear dynamics can be rewritten as a general nonlinear form:

$$
\begin{aligned}
& \dot{x}(t)=f(x)+g(x) u(t)+B_{a}, \\
& y(t)=h(x),
\end{aligned}
$$

where $f(x)=A x(t)-B_{c} x_{2}^{2}(t), g(x)=\left[g_{1}(x), \quad g_{2}(x)\right]=$ $B, u(t)=F(t), B_{a}$ is given in (10) and $h(x)=$ $\left[h_{1}(x), h_{2}(x)\right]^{T}=\left[x_{2}(t), \quad x_{6}(t)\right]^{T}$.

The vector relative degree $\left\{\rho_{1}=2, \rho_{2}=1\right\}$ can be obtained from $L_{g_{1}} h_{1}(x)=0, L_{g_{2}} h_{1}(x)=0, L_{g_{1}} L_{f} h_{1}(x)=d_{11} m_{2} \neq$ $0, L_{g_{1}} h_{2}(x)=0$, and $L_{g_{2}} h_{2}(x)=m_{3} \neq 0$. Further, it can be calculated:

$$
\begin{aligned}
\mathcal{A}(x) & \triangleq\left[\begin{array}{cc}
L_{g_{1}} L_{f}^{\rho_{1}-1} h_{1}(x) & L_{g_{2}} L_{f}^{\rho_{1}-1} h_{1}(x) \\
L_{g_{1}} L_{f}^{\rho_{2}-1} h_{2}(x) & L_{g_{2}} L_{f}^{\rho_{2}-1} h_{2}(x)
\end{array}\right] \\
& =\left[\begin{array}{cc}
d_{11} m_{2} & 0 \\
0 & m_{3}
\end{array}\right],
\end{aligned}
$$

is nonsingular for all $x$.

$$
A=\left[\begin{array}{cccccccc}
0 & 1 & 0 & 0 & 0 & 0 & 0 & 0 \\
0 & -d_{11}-b_{1} & -k_{11} & d_{11} & 0 & 0 & 0 & 0 \\
0 & 1 & 0 & -1 & 0 & 0 & 0 & 0 \\
0 & d_{12} & k_{12} & -d_{12}-d_{22}-b_{2} & -k_{22} & d_{22} & 0 & 0 \\
0 & 0 & 0 & 1 & 0 & -1 & 0 & 0 \\
0 & 0 & 0 & d_{23} & k_{23} & -d_{23}-d_{33}-b_{3} & -k_{33} & d_{33} \\
0 & 0 & 0 & 0 & 0 & 1 & 0 & -1 \\
0 & 0 & 0 & 0 & 0 & d_{34} & k_{34} & -d_{34}-b_{4}
\end{array}\right],
$$


Under the relative degree $\left\{\rho_{1}, \rho_{2}\right\}$, differentiating $y_{1}$ and $y_{2}$ in (12)-(13) or (7)-(11), with respect to time, $\rho_{1}$ and $\rho_{2}$ times, respectively, we obtain

$$
\begin{aligned}
\ddot{y}_{1}(t)= & -\left(d_{11}+b_{1}\right) \dot{x}_{2}(t)-k_{11} x_{2}(t)+k_{11} x_{4}(t) \\
& -c_{1} 2 x_{2}(t) \dot{x}_{2}(t)+d_{11}\left(d_{12} x_{2}(t)+k_{12} x_{3}(t)\right. \\
& \left.-\left(d_{12}+d_{22}+b_{2}\right) x_{4}(t)\right)-k_{22} x_{5}(t)+d_{22} x_{6}(t) \\
& +d_{11}\left(a_{2}+m_{2} F_{2}(t)\right) \\
\dot{y}_{2}(t)= & d_{23} x_{4}(t)+k_{23} x_{5}(t)-\left(d_{23}+d_{33}+b_{3}\right) x_{6}(t) \\
& -k_{33} x_{7}(t)+d_{33} x_{8}(t)+m_{3} F_{3}(t)+a_{3}
\end{aligned}
$$

for which the feedback linearization can be designed. It should be pointed out that the variable $\dot{x}_{2}(t)$ is the acceleration of the 1 st car, which is measurable and can be used in the controller design directly.

\section{B. Feedback Linearization}

If the parameters $d_{p q}, k_{p q}, m_{p}$ and $a_{p}$ for $p, q=1,2,3,4$ in the dynamics (15)-(16) are known, with the nonsingular $\mathcal{A}(x)$ in (14), we can design the linearizing control laws,

$$
\begin{aligned}
F_{2}(t)= & -\frac{1}{d_{11} m_{2}}\left(-\left(d_{11}+b_{1}\right) \dot{x}_{2}(t)-k_{11} x_{2}(t)+k_{11} x_{4}(t)\right. \\
& -c_{1} 2 x_{2}(t) \dot{x}_{2}(t)+d_{11} a_{2}+d_{11}\left(d_{12} x_{2}(t)+k_{12} x_{3}(t)\right. \\
& \left.-\left(d_{12}+d_{22}+b_{2}\right) x_{4}(t)-k_{22} x_{5}(t)+d_{22} x_{6}(t)\right) \\
& \left.-\nu_{2}(t)\right), \\
F_{3}(t)= & -\frac{1}{m_{3}}\left(d_{23} x_{4}(t)+k_{23} x_{5}(t)-\left(d_{23}+d_{33}+b_{3}\right) x_{6}(t)\right. \\
& \left.-k_{33} x_{7}(t)+d_{33} x_{8}(t)+a_{3}-\nu_{3}(t)\right) .
\end{aligned}
$$

Applying (17) and (18) to (15) and (16), respectively, we obtain the linearized system as

$$
\begin{aligned}
& \ddot{y}_{1}(t)=\nu_{2}(t), \\
& \dot{y}_{2}(t)=\nu_{3}(t),
\end{aligned}
$$

where $\nu_{2}(t)$ and $\nu_{3}(t)$ are linear feedback control laws to be designed to stabilize the linearized system (19)-(20).

We have obtained the linearization controllers $F_{2}(t)$ and $F_{3}(t)$. If the parameters $d_{p q}, k_{p q}, m_{p}$ and $a_{p}$ for $p, q=$ $1,2,3,4$ in the dynamics (15)-(16) are unknown, adaptive controllers need to be designed to replace $F_{2}(t)$ and $F_{3}(t)$. To design the adaptive controller, the stability performance for the whole system should be analyzed. The coordinate transformation (diffeomorphism) will be introduced with the feedback linearization controller to obtain a normal form for controller design and stability analysis.

\section{Normal Form}

According to the relative degree $\left\{\rho_{1}, \rho_{2}\right\}$, the 3 partial coordinate functions to transform the nonlinear system (12)(13) into a normal form, can be set as

$$
\begin{aligned}
& \xi_{1}(t)=\phi_{1}(x)=h_{1}(x)=x_{2}(t), \\
& \xi_{2}(t)=\phi_{2}(x)=L_{f} h_{1}(x)=-\left(d_{11}+b_{1}\right) x_{2}(t)-k_{11} x_{3}(t) \\
& +d_{11} x_{4}(t)-c_{1} x_{2}^{2}(t)+a_{1},(22) \\
& \xi_{3}(t)=\phi_{3}(x)=h_{2}(t)=x_{6}(t) .
\end{aligned}
$$

Then, we should find the remaining 5 partial coordinate functions $\phi_{4}(x), \phi_{5}(x), \phi_{6}(x), \phi_{7}(x), \phi_{8}(x)$, to complete the transformation such that $L_{g_{i}} \phi_{4}(x)=L_{g_{i}} \phi_{6}(x)=L_{g_{i}} \phi_{6}(x)=$ $L_{g_{i}} \phi_{7}(x)=L_{g_{i}} \phi_{8}(x)=0$, for $i=1,2$, by taking

$$
\begin{array}{ll}
\eta_{1}(t)=\phi_{4}(x)=x_{1}(t), & \eta_{2}(t)=\phi_{5}(x)=x_{3}(t), \\
\eta_{3}(t)=\phi_{6}(x)=x_{5}(t), & \eta_{4}(t)=\phi_{7}(x)=x_{7}(t), \\
\eta_{5}(t)=\phi_{4}(x)=x_{8}(t) . &
\end{array}
$$

Further, it can be calculated that the Jacobian matrix of the transformation $\Phi(x)=\left[\phi_{1}(x), \cdots, \phi_{8}(x)\right]^{T}$ is nonsingular for all $x$, and the inverse transformation is given by

$$
\begin{aligned}
x_{1}(t)= & \eta_{1}(t), \quad x_{2}(t)=\xi_{1}(t), \quad x_{3}(t)=\eta_{2}(t), \\
x_{4}(t)= & \frac{d_{11}+b_{1}}{d_{11}} \xi_{1}(t)+\frac{1}{d_{11}} \xi_{2}(t)+\frac{k_{11}}{d_{11}} \eta_{2}(t)+\frac{c_{1}}{d_{11}} \xi_{1}^{2}(t) \\
& -\frac{1}{d_{11}} a_{1}, \quad x_{5}(t)=\eta_{3}(t), \\
x_{6}(t)= & \xi_{3}(t), \quad x_{7}(t)=\eta_{4}(t), \quad x_{8}(t)=\eta_{5}(t) .
\end{aligned}
$$

Then, in these new coordinates (21)-(25), with the linearizing control laws (17) and (18), the the high-speed train dynamic system (7) can be transformed into two subsystems: the control dynamics subsystem

$$
\begin{aligned}
& \dot{\xi}_{1}(t)=\xi_{2}(t), \\
& \dot{\xi}_{2}(t)=\nu_{2}(t), \\
& \dot{\xi}_{3}(t)=\nu_{3}(t),
\end{aligned}
$$

and the zero dynamic subsystem

$$
\begin{aligned}
\dot{\eta}_{1}(t)= & \xi_{1}(t) \\
\dot{\eta}_{2}(t)= & -\frac{k_{11}}{d_{11}} \eta_{2}(t)-\frac{b_{1}}{d_{11}} \xi_{1}(t)-\frac{1}{d_{11}} \xi_{2}(t)-\frac{c_{1}}{d_{11}} \xi_{1}^{2}(t) \\
& +\frac{a_{1}}{d_{11}}, \\
\dot{\eta}_{3}(t)= & \frac{k_{11}}{d_{11}} \eta_{2}(t)+\frac{d_{11}+b_{1}}{d_{11}} \xi_{1}(t)+\frac{1}{d_{11}} \xi_{2}(t)+\frac{c_{1}}{d_{11}} \xi_{1}^{2}(t) \\
& -\xi_{3}(t)-\frac{a_{1}}{d_{11}}, \\
\dot{\eta}_{4}(t)= & -\eta_{5}(t)+\xi_{3}(t), \\
\dot{\eta}_{5}(t)= & k_{34} \eta_{4}(t)-\left(d_{34}+b_{4}\right) \eta_{5}(t)+d_{34} \xi_{3}(t)+a_{4}
\end{aligned}
$$

in which (32)-(36) is completely decoupled from the input signal, and $\xi_{1}(t)=x_{2}(t)=\dot{z}_{1}(t), \xi_{3}(t)=x_{6}(t)=\dot{z}_{3}(t)$ are the speeds of the 1 st and 3rd cars, respectively.

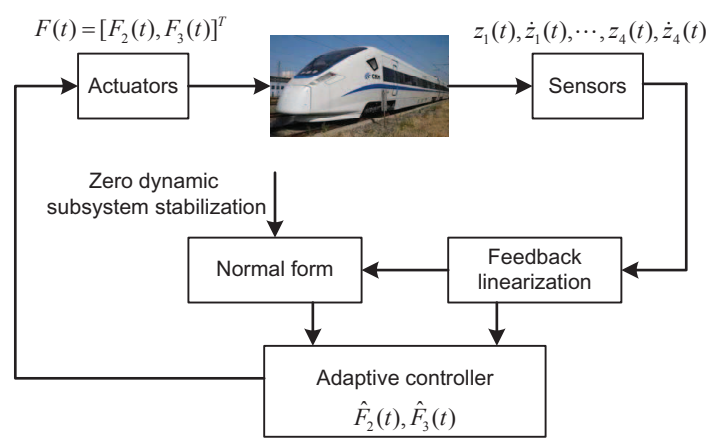

Fig. 2: Framework of the proposed adaptive control system 
So far, we have obtained the linearized normal form of the train dynamic system (7), which contains a control dynamics subsystem and a zero dynamics subsystem. To achieve the tracking performance, the stability analysis should be carried out for the zero dynamics subsystem (32)-(36) to help the adaptive controller design. Then, the framework of the proposed adaptive control can be implemented as Fig. 2, in which the designed adaptive controllers $\hat{F}_{2}(t)$ and $\hat{F}_{3}(t)$ replace $F_{2}(t)$ and $F_{3}(t)$ to control the train.

\section{System Zero Dynamics Analysis}

Zero dynamics is an important concept for nonlinear control systems, which describes some "internal" behaviors of the systems [21]. The stability performance of zero dynamics could influence the effectiveness of the designed adaptive controller. In this section, the detailed stability analysis for the zero dynamics subsystem (32)-(36) will be studied, and the stabilization condition will also be derived.

\section{A. Zero Dynamic System}

Since the terms $\frac{1}{d_{11}} a_{1}$ and $a_{4}$ in (33), (34) and (36) are constants, then $\eta(t) \stackrel{d_{11}}{=}\left[\eta_{1}(t), \quad \eta_{2}(t), \quad \eta_{3}(t), \quad \eta_{4}(t), \eta_{5}(t)\right]^{T}$,

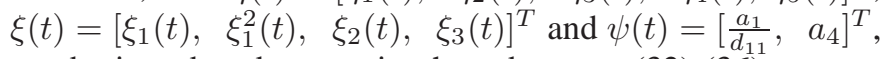
can be introduced to rewrite the subsystem (32)-(36) as

$$
\dot{\eta}(t) \triangleq A_{1} \eta(t)+B_{1} \xi(t)+B_{2} \psi(t),
$$

where

$$
\begin{aligned}
A_{1} & =\left[\begin{array}{ccccc}
0 & 0 & 0 & 0 & 0 \\
0 & -\frac{k_{11}}{d_{11}} & 0 & 0 & 0 \\
0 & \frac{k_{11}}{d_{11}} & 0 & 0 & 0 \\
0 & 0 & 0 & 0 & -1 \\
0 & 0 & 0 & k_{34} & -\left(d_{34}+b_{4}\right)
\end{array}\right], \\
B_{1}= & {\left[\begin{array}{cccc}
1 & 0 & 0 & 0 \\
-\frac{b_{1}}{d_{11}} & -\frac{c_{1}}{d_{11}} & -\frac{1}{d_{11}} & 0 \\
\frac{d_{11}+b_{1}}{d_{11}} & \frac{c_{1}}{d_{11}} & \frac{1}{d_{11}} & -1 \\
0 & 0 & 0 & 1 \\
0 & 0 & 0 & d_{34}
\end{array}\right], B_{2}=\left[\begin{array}{cc}
0 & 0 \\
1 & 0 \\
-1 & 0 \\
0 & 0 \\
0 & 1
\end{array}\right] . }
\end{aligned}
$$

The dynamics of the subsystem (37) are called zero dynamics driven by $\xi(t)$ and $\psi(t)$, the state vector of (29)-(31) and the constant signal. We will analyze the Lyapunov stability and input-to-state stability of the zero dynamics (37), to develop a set of conditions to ensure that the desired adaptive control performance is guaranteed.

Stability definitions. The original input-to-state stability requires that with the initial condition $\eta(0), \eta(t)$ goes to zero as $t$ goes to infinity [32]. Since the system (37) is a linear system, a weak input-to-state stability concept will be introduced: $\eta(t)$ is bounded for bounded initial conditions $\eta(0)$ and bounded inputs $\xi(t)$ and $\psi(t)$. This will be characterized by the Lyapunov stability of $\dot{\eta}=A_{1} \eta$ and the bounded-inputbounded-state stability of $\dot{\eta}(t)=A_{1} \eta(t)+B_{1} \xi(t)+B_{2} \psi(t)$. We will first establish the desired Lyapunov stability for $\dot{\eta}=A_{1} \eta$, while the input-to-state stability will be studied based on the bounded-input-bounded-state stability of $\dot{\eta}(t)=$ $A_{1} \eta(t)+B_{1} \xi(t)+B_{2} \psi(t)$.

\section{B. Lyapunov Stability}

For the system (37), the signal $\psi(t)$ is considered as an extra input. To check the Lyapunov stability for (37), we should set $\xi(t)=0$ and $\psi(t)=0$. Then, the characteristic polynomial of (37) can be calculated:

$$
P(\lambda)=\frac{1}{d_{11}} \lambda^{2}\left(d_{11} \lambda+k_{11}\right)\left(\lambda^{2}+b_{4} \lambda+d_{34} \lambda+k_{34}\right) .
$$

From (39), the matrix $A_{1}$ in (37) has two zero eigenvalues, corresponding to the dynamic equations

$$
\begin{aligned}
\dot{\eta}_{1}(t) & =0, \quad \text { i.e., } \quad \dot{x}_{1}(t)=\dot{z}_{1}(t)=0, \\
\dot{\eta}_{2}(t)+\dot{\eta}_{3}(t) & =-\frac{k_{11}}{d_{11}} \eta_{2}(t)+\frac{k_{11}}{d_{11}} \eta_{2}(t)=0, \\
\text { i.e., } \quad \dot{x}_{3}(t)-\dot{x}_{5}(t) & =\dot{z}_{1}(t)-\dot{z}_{4}(t)=0,
\end{aligned}
$$

which implies

$$
\begin{aligned}
\eta_{1}(t) & =z_{1}(t)=z_{1}(0), \\
\eta_{2}(t)+\eta_{3}(t) & =z_{1}(t)-z_{4}(t)=z_{1}(0)-z_{4}(0),
\end{aligned}
$$$$
\text { i.e., } z_{4}(t)=z_{4}(0) \text {. }
$$

From (42) and (43), the the dynamics (37) corresponding to the two zero eigenvalues are stable. Further, the zeros of $\left(d_{11} \lambda+k_{11}\right)\left(\lambda^{2}+b_{4} \lambda+d_{34} \lambda+k_{34}\right)$ having negative real parts means that the other three non-zero eigenvalues of $A_{1}$ are stable. Hence, we can conclude that the zero dynamic (37) is Lyapunov stable.

Then, the following result can be obtained directly.

\section{Lemma 1: The zero dynamic (37) is Lyapunov stable.}

Since the the zero dynamic system (37) is linear and timeinvariant, Lemma 1 is equivalent to the fact that the solution $\eta(t)$ of $\dot{\eta}=A_{1} \eta$ is bounded for any $\eta(0) \neq 0$.

\section{Partial Input-to-State Stability (ISS) Analysis}

The objective now is to analyze the input-to-state stability of system (37) with $\xi(t)$ and $\psi(t)$ as inputs. It should be noted that for matrix $A_{1}$, all the elements in the first row and first column are zero.

For $\xi_{1}(t)$ and $\eta_{1}(t)$ representing the speed and position of the first body, the control objective is to achieve desired speed tracking, that is, the speed $\xi_{1}(t)=\dot{z}_{1}(t)$ tracks the desired speed trajectory, while the position $\eta_{1}(t)=z_{1}(t)$ may be unbounded, that is, $\lim _{t \rightarrow \infty} \eta_{1}(t)=\infty$. Hence, in the analysis of the bounded-input-bounded-state (bounded-output) stability of (37), the state variable $\eta_{1}(t)$ is separated from the rest of the state variables in $\eta(t)$ and only the boundedness of the partial state vector $\bar{\eta}(t)=\left[\eta_{2}(t), \eta_{3}(t), \eta_{4}(t), \eta_{5}(t)\right]^{T}$ is considered. Thus, for the zero dynamics system (37), we study the partial input-to-state stability, due to the perhaps unbounded $\eta_{1}(t)$.

Eliminating the state $\eta_{1}(t)$ from (37), the following transfer function matrix from $\xi$ and $\psi$ to $\bar{\eta}$ can be calculated:

$$
\bar{\eta}(s)=\bar{G}_{1}(s) \xi(s)+\bar{G}_{2}(s) \psi(s),
$$


where

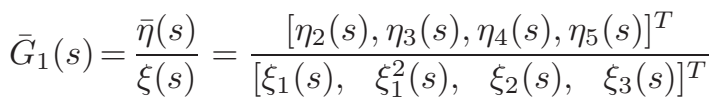

$$
\begin{aligned}
& =\frac{1}{P(s)}\left[\begin{array}{cccc}
Z_{11}(s) & Z_{12}(s) & Z_{13}(s) & Z_{14}(s) \\
Z_{21}(s) & Z_{22}(s) & Z_{23}(s) & Z_{24}(s) \\
Z_{31}(s) & Z_{32}(s) & Z_{33}(s) & Z_{34}(s) \\
Z_{41}(s) & Z_{42}(s) & Z_{43}(s) & Z_{44}(s)
\end{array}\right] \\
& \bar{G}_{2}(s)=\frac{\bar{\eta}(s)}{\varphi(s)}=\frac{\left[\eta_{2}(s), \eta_{3}(s), \eta_{4}(s), \eta_{5}(s)\right]^{T}}{\left[\frac{a_{1}}{d_{11}}(s), a_{4}(s)\right]^{T}} \\
& =\left[\begin{array}{cc}
\frac{d_{11}}{d_{11} s+k_{11}} & 0 \\
\frac{-d_{11}}{d_{11} s+k_{11}} & 0 \\
0 & \frac{-1}{s^{2}+\left(d_{34}+b_{4}\right) s+k_{34}} \\
0 & \frac{s}{s^{2}+\left(d_{34}+b_{4}\right) s+k_{34}}
\end{array}\right],
\end{aligned}
$$

with the elements in (45) being given as

$$
\begin{aligned}
P(s) & =\frac{1}{d_{11}} s\left(d_{11} s+k_{11}\right)\left(s^{2}+b_{4} s+d_{34} s+k_{34}\right), \\
Z_{11}(s) & =-\frac{b_{1}}{d_{11}} s\left(s^{2}+b_{4} s+d_{34} s+k_{34}\right), \\
Z_{12}(s) & =-\frac{c_{1}}{d_{11}} s\left(s^{2}+b_{4} s+d_{34} s+k_{34}\right), \\
Z_{13}(s) & =-\frac{1}{d_{11}} s\left(s^{2}+b_{4} s+d_{34} s+k_{34}\right), \quad Z_{14}(s)=0, \\
Z_{21}(s) & =\frac{1}{d_{11}}\left(b_{1} s+d_{11} s+k_{11}\right)\left(s^{2}+b_{4} s+d_{34} s+k_{34}\right), \\
Z_{22}(s) & =\frac{c_{1}}{d_{11}} s\left(s^{2}+b_{4} s+d_{34} s+k_{34}\right), \\
Z_{23}(s) & =\frac{1}{d_{11}} s\left(s^{2}+b_{4} s+d_{34} s+k_{34}\right), \\
Z_{24}(s) & =-\frac{1}{d_{11}}\left(d_{11} s+k_{11}\right)\left(s^{2}+b_{4} s+d_{34} s+k_{34}\right), \\
Z_{31}(s) & =0, \quad Z_{32}(s)=0, \quad Z_{33}(s)=0, \\
Z_{34}(s) & =\frac{1}{d_{11}} s\left(d_{11} s+k_{11}\right)\left(s+b_{4}\right), \\
Z_{41}(s) & =0, \quad Z_{42}(s)=0, \quad Z_{43}(s)=0, \\
Z_{44}(s) & =\frac{1}{d_{11}} s\left(d_{11} s+k_{11}\right)\left(d_{34} s+k_{34}\right) .
\end{aligned}
$$

Remark 2: It should be noted that a special case of $\xi_{1}(t)-$ $\xi_{3}(t) \in L^{1}$ is $\lim _{t \rightarrow \infty}\left(\xi_{1}(t)-\xi_{3}(t)\right)=0$ exponentially for Corollary 1 . Thus, if the nominal operating condition $\xi_{1}(t)=$

\begin{tabular}{|c|c|c|c|}
\hline Symbol & Description & Unit & Value \\
\hline$M_{1}$ & 1st car mass & ton & 50 \\
\hline$M_{2}$ & 2nd car mass & ton & 48 \\
\hline$M_{3}$ & 3rd car mass & ton & 51 \\
\hline$M_{4}$ & 4th car mass & ton & 53 \\
\hline$a_{1}, a_{4}$ & $\begin{array}{l}\text { mechanical resistance } \\
\text { coefficients }\end{array}$ & Ns/ton & 6.63 \\
\hline$a_{2}, a_{3}$ & $\begin{array}{l}\text { mechanical resistance } \\
\text { coefficients }\end{array}$ & Ns/ton & 5.43 \\
\hline$b_{1}, b_{4}$ & $\begin{array}{l}\text { mechanical resistance } \\
\text { coefficients }\end{array}$ & $\mathrm{N} \mathrm{s} /(\mathrm{m}$ ton $)$ & 0.06382 \\
\hline$b_{2}, b_{3}$ & $\begin{array}{l}\text { mechanical resistance } \\
\text { coefficients }\end{array}$ & $\mathrm{N} \mathrm{s} /(\mathrm{m}$ ton $)$ & 0.07041 \\
\hline$c$ & $\begin{array}{l}\text { aerodynamic resistance } \\
\text { coefficient }\end{array}$ & $\mathrm{N} \mathrm{s}^{2} /\left(\mathrm{m}^{2}\right.$ ton $)$ & 0.00107 \\
\hline$k_{1}, k_{3}$ & spring coefficients & $\mathrm{N} / \mathrm{m}$ & $500 \times 10^{6}$ \\
\hline$k_{2}$ & spring coefficient & $\mathrm{N} / \mathrm{m}$ & $650 \times 10^{6}$ \\
\hline$d_{1}, d_{3}$ & damper coefficients & $\mathrm{Ns} / \mathrm{m}$ & $2 \times 10^{4}$ \\
\hline$d_{2}$ & damper coefficient & $\mathrm{Ns} / \mathrm{m}$ & $2.2 \times 10^{4}$ \\
\hline
\end{tabular}
$\xi_{3}(t)$ or $\lim _{t \rightarrow \infty}\left(\xi_{1}(t)-\xi_{3}(t)\right)=0$ exponentially, or a relaxed operating condition $\xi_{1}(t)-\xi_{3}(t) \in L^{1}$ can be satisfied, the states $\eta_{2}(t), \eta_{3}(t), \eta_{4}(t), \eta_{5}(t)$, (i.e., $z_{1}(t)-z_{2}(t), z_{2}(t)-$ $\left.z_{3}(t), z_{3}(t)-z_{4}(t), \dot{z}_{4}(t)\right)$ of the zero dynamics (37) can be bounded and $\eta_{1}(t)=z_{1}(t)$ has the position performance.

\section{Simulation Verification}

We have studied and established the key condition for the zero dynamic system to be Lyapunov stable and partial bounded-input-bounded-state stable (see Lemmas 1 and 2, and Corollary 1). Now, we will use a real $\mathrm{CRH}_{5}$ high-speed train model from [29] to illustrate the effectiveness of the proposed stabilization condition, where the general resistance and intrain force curves are shown in Fig. 3.

TABLE I: $\mathrm{CRH}_{5}$ train parameters

The parameters in the simulation are as table I. Here, we consider the two stabilization conditions: (i) ideal case is the speeds of the 1 st and 3 rd cars are synchronous, i.e., $\dot{z}_{1}(t)=$ $\dot{z}_{3}(t)\left(\xi_{1}(t)=\xi_{3}(t)\right)$; and (ii) relaxed case is the speeds of 1 st and 3 rd cars satisfy $L_{1}$ performance, i.e., $\dot{z}_{1}(t)-\dot{z}_{3}(t) \in L^{1}$

Moreover, it can be verified from (37) that for $\xi_{1}(t) \neq \xi_{3}(t)$, $\eta_{3}(s)=\frac{Z_{21}(s) \xi_{1}(s)+Z_{22}(s) \xi_{1}^{2}(s)+Z_{23}(s) \xi_{2}(s)+Z_{24}(s) \xi_{3}(s)}{P(s)}$. Then, 
$\left(\xi_{1}(t)-\xi_{3}(t) \in L^{1}\right)$. To make $\dot{z}_{1}(t)-\dot{z}_{3}(t) \in L^{1}$, an additional signal $0.005 \exp (-0.2 t)$ is applied on $\dot{z}_{1}(t)$ as $\dot{z}_{1}(t)=\dot{z}_{3}(t)+$ $0.005 \exp (-0.2 t)$, which makes $\dot{z}_{1}(t)-\dot{z}_{3}(t) \in L^{1}$. Then, the simulation results are shown in figs. 4-7.

Figs. 4 and 6 show the simulation results of the speeds for the 2nd and 4th cars including the plant speed (solid) and referred speed of 3 rd car (dashed) under the ideal condition $\xi_{1}(t)=\xi_{3}(t)$ and the relaxed condition $\xi_{1}(t)-\xi_{3}(t) \in L^{1}$, respectively, in which the initial values of the 2 nd and 4th cars' speeds are $0.01 \mathrm{~m} / \mathrm{s}$. From figs. 4 and 6 , it can be seen that the speeds of the 2nd and 4th cars are bounded.

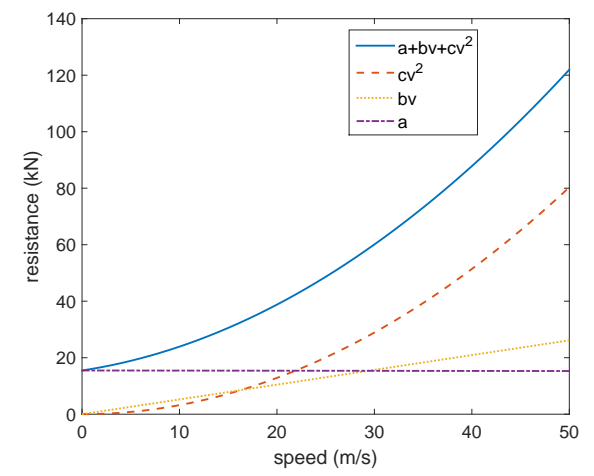

(a) Aerodynamic and mechanical components of resistances versus speed

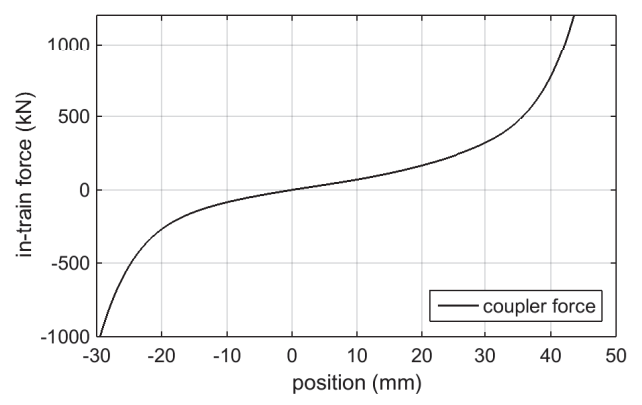

(b) Coupler Force versus position

Fig. 3: General resistance and in-train forces
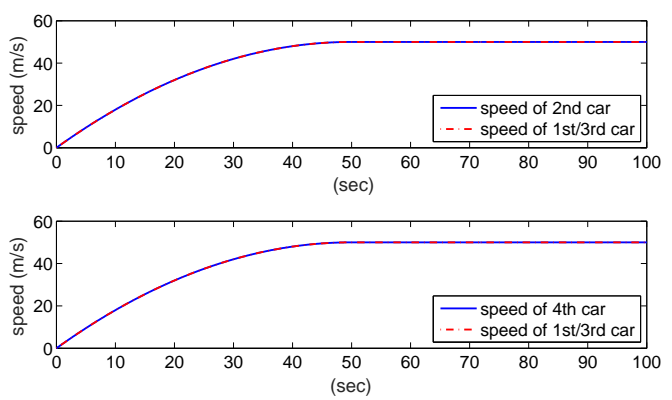

Fig. 4: Speeds of the 2nd and 4th cars under $\xi_{1}(t)=\xi_{3}(t)$ $\left(\dot{z}_{1}(t)=\dot{z}_{3}(t)\right)$

Figs. 5 and 7 show the position of the 1 st car and the relative positions between the 1 st and 2 nd cars $z_{1}(t)-z_{2}(t)$, the 2nd and 3rd cars $z_{2}(t)-z_{3}(t)$, and the 3rd and 4th cars
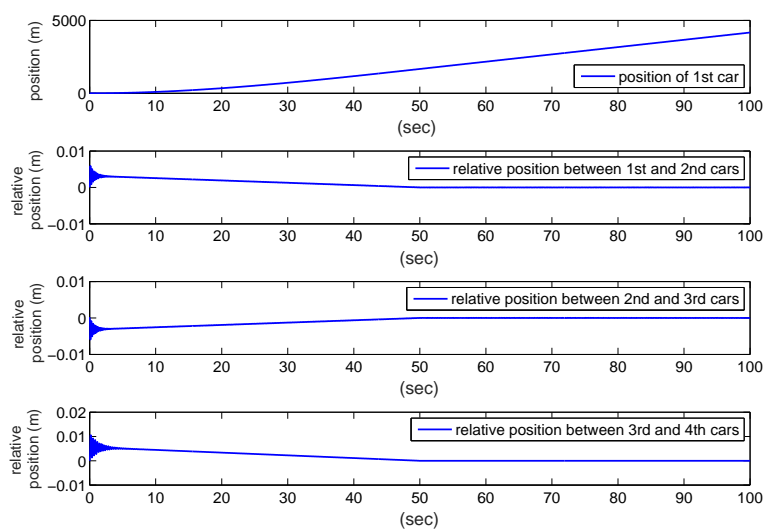

Fig. 5: Positions of cars under $\xi_{1}(t)=\xi_{3}(t)\left(\dot{z}_{1}(t)=\dot{z}_{3}(t)\right)$
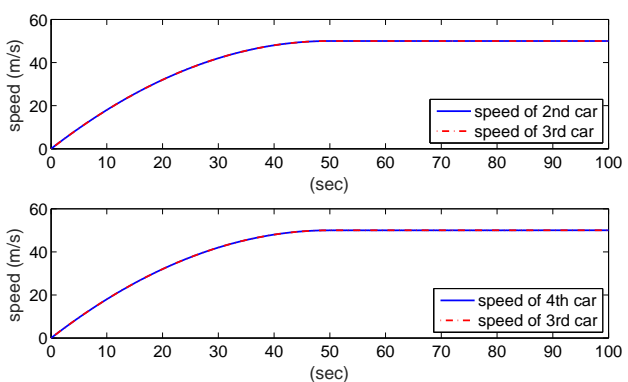

Fig. 6: Speeds of the 2nd and 4th cars under $\xi_{1}(t)-\xi_{3}(t) \in L^{1}$ $\left(\dot{z}_{1}(t)-\dot{z}_{4}(t) \in L^{1}\right)$
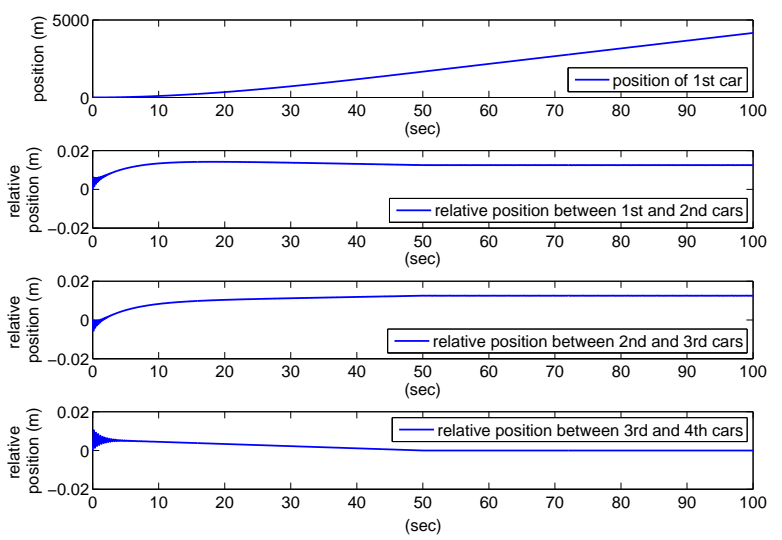

Fig. 7: Positions of cars under $\xi_{1}(t)-\xi_{3}(t) \in L^{1}$ $\left(\dot{z}_{1}(t)-\dot{z}_{4}(t) \in L^{1}\right)$

$z_{3}(t)-z_{4}(t)$, under the ideal condition $\xi_{1}(t)=\xi_{3}(t)$ and the relaxed condition $\xi_{1}(t)-\xi_{3}(t) \in L^{1}$, respectively. The relative positions become constants under the stabilization conditions, while the position of the 1st car would go infinity when the speed of the 1st does not go to zero, which is in consistence with the real case.

The simulation results show that the proposed stabilization conditions can achieve the boundedness of the speeds and the relative positions, and the position of the 1st car satisfy the 
performance. Then, in the next section, we will design a nominal control scheme to ensure that $\lim _{t \rightarrow \infty}\left(\xi_{1}(t)-\xi_{3}(t)\right)=$ 0 exponentially, and an adaptive control scheme to ensure $\xi_{1}(t)-\xi_{3}(t) \in L^{1}$, so that the closed-loop control system is stable and asymptotic output speed tracking is achieved.

\section{Speed Tracking Control Schemes}

Considering the speed tracking task for the train, we choose the desired speed trajectory $v_{m}(t)$. Besides the stabilization condition proposed in Corollary 1 , the controller should be designed such that the states $\xi_{1}(t), \xi_{2}(t)$ and $\xi_{3}(t)$ are bounded, and the speed tracking performance is achieved, i.e., $\lim _{t \rightarrow \infty}\left(\xi_{1}(t)-v_{m}(t)\right)=0$ and $\lim _{t \rightarrow \infty}\left(\xi_{3}(t)-v_{m}(t)\right)=0$.

Moreover, if $\lim _{t \rightarrow \infty}\left(\xi_{1}(t)-v_{m}(t)\right)=0$ exponentially and $\lim _{t \rightarrow \infty}\left(\xi_{3}(t)-v_{m}(t)\right)=0$ exponentially, then $\lim _{t \rightarrow \infty}\left(\xi_{1}(t)-\xi_{3}(t)\right)=0$ exponentially; and if $\xi_{1}(t)-$ $v_{m}(t) \in L^{1}$ and $\xi_{3}(t)-v_{m}(t) \in L^{1}$, then $\xi_{1}(t)-\xi_{3}(t) \in L^{1}$. The stabilization condition proposed in Corollary 1 can be equivalent to the new one that $\lim _{t \rightarrow \infty}\left(\xi_{1}(t)-v_{m}(t)\right)=0$ exponentially and $\lim _{t \rightarrow \infty}\left(\xi_{3}(t)-v_{m}(t)\right)=0$ exponentially, or $\xi_{1}(t)-v_{m}(t) \in L^{1}$ and $\xi_{3}(t)-v_{m}(t) \in L^{1}$.

In this section, the nominal controller will be presented to make $\lim _{t \rightarrow \infty}\left(\xi_{1}(t)-v_{m}(t)\right)=0$ exponentially and $\lim _{t \rightarrow \infty}\left(\xi_{3}(t)-v_{m}(t)\right)=0$ exponentially, and the adaptive controller will be proposed to ensure $\xi_{1}(t)-v_{m}(t) \in L^{1}$, $\xi_{3}(t)-v_{m}(t) \in L^{1}, \lim _{t \rightarrow \infty}\left(\xi_{1}(t)-v_{m}(t)\right)=0$ and $\lim _{t \rightarrow \infty}\left(\xi_{3}(t)-v_{m}(t)\right)=0$, in the presence of the unknown system parameters $d_{p q}, k_{p q}, m_{p}$ and $a_{p}$ for $p, q=1,2,3,4$. Then, from the nominal and adaptive closed-loop control systems, the overall system stability analysis will be derived.

\section{A. Nominal Control Schemes}

If the parameters $d_{p q}, k_{p q}, m_{p}$ and $a_{p}$ for $p, q=1,2,3,4$ in the dynamics (15)-(16) are known, the linearizing control laws (17) and (18) can be used directly with the coordinates (21)-(25) to obtain the linearized system (29)-(36). Then, for the nominal controllers (17) and (18), the main task is to design the control signals $\nu_{2}(t)$ and $\nu_{3}(t)$ to achieve that $\lim _{t \rightarrow \infty}\left(\xi_{1}(t)-v_{m}(t)\right)=0$ exponentially and $\lim _{t \rightarrow \infty}\left(\xi_{3}(t)-v_{m}(t)\right)=0$ exponentially.

Output tracking control $\nu_{2}(t)$ design. According to the coordinate transformations (21)-(23), it has $\xi_{1}(t)=x_{2}(t)$ and $\xi_{3}(t)=x_{6}(t)$. Then, the controller design for the dynamics $\xi_{1}(t)$ and $\xi_{3}(t)$ is equivalent to that for the dynamics $x_{1}(t)$ and $x_{6}(t)$.

The dynamics system (29)-(30) can be rewritten as

$$
\ddot{\xi}_{1}(t)=\ddot{x}_{2}(t)=\nu_{2}(t),
$$

where $x_{2}(t)=\dot{z}_{1}(t)$ is the speed of the 1 st car, the control signal $\nu_{2}(t)$ is proposed as

$$
\begin{aligned}
\nu_{2}(t)= & \ddot{v}_{m}(t)-\alpha_{1}\left(\dot{x}_{2}(t)-\dot{v}_{m}(t)\right) \\
& -\alpha_{2}\left(x_{2}(t)-v_{m}(t)\right),
\end{aligned}
$$

with $\alpha_{1}>0$ and $\alpha_{2}>0$ being design parameters such that $s^{2}+\alpha_{1} s+\alpha_{2}$ is a Hurwitz polynomial. It should be noted that the desired speed $v_{m}(t)$, the acceleration $\dot{v}_{m}(t)$ and its derivative $\ddot{v}_{m}(t)$ are bounded.

Submitting $\nu_{2}(t)$ into the system (50), it has

$$
\begin{gathered}
\ddot{x}_{2}(t)-\ddot{v}_{m}(t)=-\alpha_{1}\left(\dot{x}_{2}(t)-\dot{v}_{m}(t)\right) \\
-\alpha_{2}\left(x_{2}(t)-v_{m}(t)\right) .
\end{gathered}
$$

With the tracking error $e_{2}(t)=x_{2}(t)-v_{m}(t)=\xi_{1}(t)-$ $v_{m}(t),(52)$ leads to

$$
\ddot{e}_{2}(t)+\alpha_{1} \dot{e}_{2}(t)+\alpha_{2} e_{2}(t)=0,
$$

which implies that $\lim _{t \rightarrow \infty} e_{2}(t)=\lim _{t \rightarrow \infty} \dot{e}_{2}(t)=0$ exponentially.

Output tracking control $\nu_{3}(t)$ design. The design procedure for the control signal $\nu_{3}(t)$, similar to that of $\nu_{2}(t)$, will be given. The dynamics of state $\xi_{3}(t)$ can be written as:

$$
\dot{\xi}_{3}(t)=\dot{x}_{6}(t)=\nu_{3}(t),
$$

where $x_{6}(t)=\dot{z}_{3}(t)$ is the speed of the 3rd car, the control signal $\nu_{3}(t)$ is designed as:

$$
\nu_{3}(t)=\dot{v}_{m}(t)-\alpha_{3}\left(x_{6}(t)-v_{m}(t)\right),
$$

with $\alpha_{3}>0$ a design constant.

For (54), the signal (55) leads to that the tracking error $e_{6}(t)=x_{6}(t)-v_{m}(t)=\xi_{3}(t)-v_{m}(t)$ satisfies:

$$
\dot{e}_{6}(t)+\alpha_{3} e_{6}(t)=0,
$$

which implies that $\lim _{t \rightarrow \infty} e_{6}(t)=0$ exponentially.

Remark 3: For the relative degree 2 subsystem (50), the acceleration $\dot{x}_{2}(t)\left(\ddot{z}_{1}(t)\right)$ of the 1 st car is used in the output tracking controller $\nu_{2}(t)$ design. In practice, the accelerations of these cars are usually measurable. So, the proposed nominal controllers $F_{2}(t)$ and $F_{3}(t)$ can be implemented for a real train, when the system parameters are known.

\section{B. Adaptive Control Design for $F_{2}(t)$}

As the system parameters $d_{11}, m_{2}, d_{12}, k_{11}, b_{1}, c_{1}, k_{12}, d_{22}$, $b_{2}$, and $k_{22}$ are unknown, an adaptive controller $\hat{F}_{2}(t)$ instead of the nominal controller $F_{2}(t)$ should be designed, such that $\xi_{1}(t)-v_{m}(t) \in L^{1}$ and $\lim _{t \rightarrow \infty}\left(\xi_{1}(t)-v_{m}(t)\right)=0$.

Adaptive controller structure. Since there is no unknown parameter in the linearized system (50) or (29)-(30), the output tracking control $\nu_{2}(t)$ in (51) can be used directly in the adaptive controller design. To design the adaptive controller $\hat{F}_{2}(t)$, the parameters of the nominal controller $F_{2}(t)$ in (17) are defined:

$$
\begin{aligned}
& \theta_{11}=\frac{1}{d_{11} m_{2}}, \quad \theta_{12}=d_{11} d_{12}-k_{11}, \quad \theta_{13}=d_{11}+b_{1}, \\
& \theta_{14}=c_{1}, \quad \theta_{15}=d_{11} k_{12}, \quad \theta_{17}=d_{11} k_{22}, \quad \theta_{18}=d_{11} d_{22}, \\
& \theta_{16}=d_{11} d_{12}+d_{11} d_{22}+d_{11} b_{2}-k_{11}, \quad \theta_{19}=d_{11} a_{2},
\end{aligned}
$$

which lead to the nominal controller $F_{2}(t)$ being written as

$$
\begin{aligned}
F_{2}(t)= & -\theta_{11}\left(\theta_{12} x_{2}(t)-\theta_{13} \dot{x}_{2}(t)-2 \theta_{14} x_{2}(t) \dot{x}_{2}(t)\right. \\
& +\theta_{15} x_{3}(t)-\theta_{16} x_{4}(t)-\theta_{17} x_{5}(t)+\theta_{18} x_{6}(t) \\
& \left.+\theta_{19}-\nu_{2}(t)\right) .
\end{aligned}
$$


Design the adaptive controller $\hat{F}_{2}(t)$ as

$$
\begin{aligned}
\hat{F}_{2}(t)= & -\hat{\theta}_{11}(t)\left(\hat{\theta}_{12}(t) x_{2}(t)-\hat{\theta}_{13}(t) \dot{x}_{2}(t)\right. \\
& -2 \hat{\theta}_{14}(t) x_{2}(t) \dot{x}_{2}(t)+\hat{\theta}_{15}(t) x_{3}(t)-\hat{\theta}_{16}(t) x_{4}(t) \\
& \left.-\hat{\theta}_{17}(t) x_{5}(t)+\hat{\theta}_{18}(t) x_{6}(t)+\hat{\theta}_{19}(t)-\nu_{2}(t)\right) \\
& +\bar{\nu}_{2}(t)
\end{aligned}
$$

where $\hat{\theta}_{1 \varrho}(t)$ are the estimations of $\theta_{1 \varrho}$, for $\varrho=1,2, \ldots, 9$, $\nu_{2}(t)$ is given in (51), and $\bar{\nu}_{2}(t)$ is a designed signal.

Closed-loop adaptive control system. To design the adaptive laws for $\hat{\theta}_{1 \varrho}(t)$, with $\varrho=1,2, \ldots, 9$, we define the parameter errors $\tilde{\theta}_{1 \varrho}(t)=\hat{\theta}_{1 \varrho}(t)-\theta_{1 \varrho}$ and use the control law (60) and the system (15) under the definition (57)-(58), to obtain

$$
\begin{aligned}
\ddot{x}_{2}(t)= & \ddot{z}_{m}(t)-\alpha_{1}\left(\dot{x}_{2}(t)-\dot{v}_{m}(t)\right)-\alpha_{2}\left(x_{2}(t)-v_{m}(t)\right) \\
& +\tilde{\theta}_{11}(t) \hat{F}_{2}(t)-\tilde{\theta}_{12}(t) x_{2}(t)+\tilde{\theta}_{13}(t) \dot{x}_{2}(t) \\
& +2 \tilde{\theta}_{14}(t) x_{2}(t) \dot{x}_{2}(t)-\tilde{\theta}_{15}(t) x_{3}(t)+\tilde{\theta}_{16}(t) x_{4}(t) \\
& +\tilde{\theta}_{17}(t) x_{5}(t)-\tilde{\theta}_{18}(t) x_{6}(t)-\tilde{\theta}_{19}(t)+d_{11} m_{2} \bar{\nu}_{2}(t),
\end{aligned}
$$

which can be rewritten as

$$
\begin{aligned}
& \ddot{e}_{2}(t)+\alpha_{1} \dot{e}_{2}(t)+\alpha_{2} e_{2}(t) \\
= & \tilde{\Theta}_{1}^{T}(t) W_{1}(t)+d_{11} m_{2} \bar{\nu}_{2}(t),
\end{aligned}
$$

with $e_{2}(t)=x_{2}(t)-v_{m}(t), x_{2}(t)=\xi_{1}(t), \tilde{\Theta}_{1}(t)=$ $\left[\tilde{\theta}_{11}(t), \tilde{\theta}_{12}(t), \ldots, \tilde{\theta}_{19}(t)\right]^{T}$ and $W_{1}(t)=\left[\hat{F}_{2}(t),-x_{2}(t)\right.$, $\left.\dot{x}_{2}(t), x_{2}(t) \dot{x}_{2}(t),-x_{3}(t), x_{4}(t), x_{5}(t),-x_{6}(t),-1\right]^{T}$.

Let $\bar{e}_{2}(t)=\left[\begin{array}{ll}e_{2}(t) & \dot{e}_{2}(t)\end{array}\right]^{T}$. Then error dynamics (61) can be recast as

$$
\begin{aligned}
\dot{\bar{e}}_{2}(t)= & {\left[\begin{array}{cc}
0 & 1 \\
-\alpha_{2} & -\alpha_{1}
\end{array}\right] \bar{e}_{2}(t) } \\
& +\left[\begin{array}{c}
0 \\
\tilde{\Theta}_{1}^{T}(t) W_{1}(t)+d_{11} m_{2} \bar{\nu}_{2}(t)
\end{array}\right] .
\end{aligned}
$$

Due to the Hurwitz polynomial $s^{2}+\alpha_{1} s+\alpha_{2}$, there exist positive definite matrices $P=P^{T}=\left[\begin{array}{ll}p_{11} & p_{12} \\ p_{12} & p_{22}\end{array}\right]>0$ and $Q=Q^{T}>0$ to satisfy

$$
\left[\begin{array}{cc}
0 & 1 \\
-\alpha_{2} & -\alpha_{1}
\end{array}\right]^{T} P+P\left[\begin{array}{cc}
0 & 1 \\
-\alpha_{2} & -\alpha_{1}
\end{array}\right]=-Q
$$

Adaptive laws. The adaptive law for $\hat{\Theta}_{1}(t)=$ $\left[\hat{\theta}_{11}, \hat{\theta}_{12}, \ldots, \hat{\theta}_{19}\right]^{T}$ and signal $\bar{\nu}_{2}(t)$ are designed as

$$
\begin{aligned}
\dot{\hat{\Theta}}_{1}(t) & =-\Gamma_{1} W_{1}(t)\left(p_{12} e_{2}(t)+p_{22} \dot{e}_{2}(t)\right), \\
\bar{\nu}_{2}(t) & =-\beta_{1} \operatorname{sign}\left(p_{12} e_{2}(t)+p_{22} \dot{e}_{2}(t)\right),
\end{aligned}
$$

where $\Gamma_{1}=\operatorname{diag}\left\{\gamma_{11}, \gamma_{12}, \ldots, \gamma_{19}\right\}>0, \gamma_{1 q}, q=1,2, \ldots, 9$, are adaptation gains, $p_{12}$ and $p_{22}$ are the elements in matrix $P, \beta_{1}>0$ is a design parameter related to the convergence rate of $x_{2}(t)-v_{m}(t)$.

Subsystem analysis. Consider the Lyapunov function

$$
V_{1}\left(\bar{e}_{2}(t), \tilde{\Theta}_{1}(t)\right)=\frac{1}{2} \bar{e}_{2}^{T}(t) P \bar{e}_{2}(t)+\frac{1}{2} \tilde{\Theta}_{1}^{T}(t) \Gamma_{1}^{-1} \tilde{\Theta}_{1}(t)(66)
$$

Then, from (62)-(65), the time derivative of $V_{1}\left(\bar{e}_{2}, \tilde{\Theta}_{1}\right)$ is

$$
\begin{aligned}
& \dot{V}_{1}\left(\bar{e}_{2}, \tilde{\Theta}_{1}\right) \\
= & \frac{1}{2} \dot{\bar{e}}_{2}^{T}(t) P \bar{e}_{2}(t)+\frac{1}{2} \bar{e}_{2}^{T}(t) P \dot{\bar{e}}_{2}(t)+\tilde{\Theta}_{1}^{T}(t) \Gamma_{1}^{-1} \dot{\tilde{\Theta}}_{1}(t) \\
= & -\bar{e}_{2}^{T}(t) Q \bar{e}_{2}(t)+\bar{e}_{2}^{T}(t) P\left[\begin{array}{c}
0 \\
1
\end{array}\right] d_{11} m_{2} \bar{\nu}_{2}(t) \\
= & -\bar{e}_{2}^{T}(t) Q \bar{e}_{2}(t)+\left(p_{12} e_{2}(t)+p_{22} \dot{e}_{2}(t)\right) d_{11} m_{2} \bar{\nu}_{2}(t) \\
= & -\bar{e}_{2}^{T}(t) Q \bar{e}_{2}(t)-d_{11} m_{2} \beta_{1}\left|p_{12} e_{2}(t)+p_{22} \dot{e}_{2}(t)\right|,
\end{aligned}
$$

where $Q=Q^{T}>0$ and $d_{11} m_{2} \beta_{1}>0$. (67) indicates that the closed-loop system consisting of (61), (64) and (65) is stable and its solutions are bounded, that is, all the variables $\bar{e}_{2}(t)$ and $\tilde{\Theta}_{1}(t)$ are bounded. (67) also implies $\bar{e}_{2}(t) \in L^{2}$ and $\left(p_{12} e_{2}(t)+p_{22} \dot{e}_{2}(t)\right) \in L^{1}$. The boundedness of the signal $W_{1}(t)$ will be analyzed in the following Subsection D.

Remark 4: It should be noted that due to the unknown boundedness of signal $W_{1}(t)$, the adaptive controller design method in [33] for relative degree 2 case, cannot be used to the controller design for system (61). The new adaptive controller (60) with adaptive law (64) and signal (65) is proposed to deal with this case, where the signal (65) is introduced to make $\left(p_{12} e_{2}(t)+p_{22} \dot{e}_{2}(t)\right) \in L^{1}$, to ensure the needed stabilization condition for the zero dynamic subsystem.

\section{Adaptive Control Design for $F_{3}(t)$}

It should be noted that the relative degrees for $y_{1}(t)$ and $y_{2}(t)$ are different ( 2 and 1 , respectively). Then, the design procedure of the adaptive controller $\hat{F}_{3}(t)$ for the dynamics $\xi_{3}(t)$ with relative degree 1 is a bit different from that of $\hat{F}_{2}(t)$ for the dynamic $\xi_{1}(t)$ with relative degree 2 .

Adaptive controller structure. According to the linearized system (54) or (30), the output tracking control $\nu_{3}(t)$ in (55) can be used directly in the adaptive controller design. To design the adaptive controller, the parameters of the nominal controller $F_{3}(t)$ in (18) are defined as

$$
\begin{aligned}
& \theta_{21}=\frac{1}{m_{3}}, \quad \theta_{22}=d_{23}, \quad \theta_{23}=k_{23}, \quad \theta_{25}=k_{33}, \\
& \theta_{24}=d_{23}+d_{33}+b_{3}, \quad \theta_{26}=d_{33}, \quad \theta_{27}=a_{4},
\end{aligned}
$$

which lead to the nominal controller $F_{3}(t)$ being written as

$$
\begin{aligned}
F_{3}(t)= & -\theta_{21}\left(\theta_{22} x_{4}(t)+\theta_{23} x_{5}(t)-\theta_{24} x_{6}(t)-\theta_{25} x_{7}(t)\right. \\
& \left.+\theta_{26} x_{8}(t)+\theta_{27}-\nu_{3}(t)\right)
\end{aligned}
$$

The adaptive controller $\hat{F}_{3}(t)$ is designed as

$$
\begin{aligned}
\hat{F}_{3}(t)= & -\hat{\theta}_{21}(t)\left(\hat{\theta}_{22}(t) x_{4}(t)+\hat{\theta}_{23}(t) x_{5}(t)-\hat{\theta}_{24}(t) x_{6}(t)\right. \\
& \left.-\hat{\theta}_{25}(t) x_{7}(t)+\hat{\theta}_{26}(t) x_{8}(t)+\hat{\theta}_{27}(t)-\nu_{3}(t)\right) \\
& -\beta_{2} \operatorname{sgn}\left(x_{6}(t)-v_{m}(t)\right),
\end{aligned}
$$

where $\hat{\theta}_{2 \varrho}(t)$ are the estimations of $\theta_{2 \varrho}$, for $\varrho=1,2, \ldots, 7$, $\nu_{3}(t)$ is given in (55), $\beta_{2}>0$ is a design parameter related to the convergence rate of $x_{6}(t)-v_{m}(t)$. 
Closed-loop adaptive control system. To design the adaptive laws for $\hat{\theta}_{2 \varrho}(t)$, with $\varrho=1,2, \ldots, 7$, we define the parameter errors $\hat{\theta}_{2 \varrho}(t)=\hat{\theta}_{2 \varrho}(t)-\theta_{2 \varrho}$. Using the control law (71), the system (16) under the definition (68)-(69) follows

$$
\begin{aligned}
\dot{x}_{6}(t)= & \dot{z}_{m}(t)-\alpha_{3}\left(x_{6}(t)-v_{m}(t)\right)+\tilde{\theta}_{21}(t) \hat{F}_{3}(t) \\
& +\left(-\tilde{\theta}_{22}(t) x_{4}(t)+\tilde{\theta}_{23}(t) x_{5}(t)+\tilde{\theta}_{24}(t) x_{6}(t)\right. \\
& \left.+\tilde{\theta}_{25}(t) x_{7}(t)-\tilde{\theta}_{26}(t) x_{8}(t)-\tilde{\theta}_{27}(t)\right) \\
& -m_{3} \beta_{2} \operatorname{sign}\left(x_{6}(t)-v_{m}(t)\right),
\end{aligned}
$$

which leads to

$$
\dot{e}_{6}(t)+\alpha_{3} e_{6}(t)=\tilde{\Theta}_{2}^{T}(t) W_{2}(t)-m_{3} \beta_{2} \operatorname{sgn}\left(e_{6}\right),
$$

with $e_{6}(t)=x_{6}(t)-v_{m}(t), x_{6}(t)=\xi_{3}(t), \tilde{\Theta}_{2}(t)=$ $\left[\tilde{\theta}_{21}(t), \tilde{\theta}_{22}(t), \ldots, \tilde{\theta}_{27}(t)\right]^{T}$ and $W_{2}(t)=\left[\hat{F}_{3}(t),-x_{4}(t)\right.$, $\left.x_{5}(t), x_{6}(t), x_{7}(t),-x_{8}(t),-1\right]^{T}$.

Adaptive laws. The update laws for $\hat{\Theta}_{2}(t)=$ $\left[\hat{\theta}_{21}, \hat{\theta}_{22}, \ldots, \hat{\theta}_{27}\right]^{T}$ are chosen as

$$
\dot{\hat{\Theta}}_{2}(t)=-\Gamma_{2} W_{2}(t) e_{6}(t),
$$

where $\Gamma_{2}=\operatorname{diag}\left\{\gamma_{21}, \gamma_{22}, \ldots, \gamma_{27}\right\}>0, \gamma_{2 q}, q=1,2, \ldots, 7$, are adaptation gains.

Subsystem analysis. For the system (73), consider the Lyapunov function

$$
V_{2}\left(e_{6}(t), \tilde{\Theta}_{2}(t)\right)=\frac{1}{2} e_{6}(t)+\frac{1}{2} \tilde{\Theta}_{2}^{T}(t) \Gamma_{2}^{-1} \tilde{\Theta}_{2}(t) .
$$

Then, the time derivative of $V_{2}\left(e_{6}, \tilde{\Theta}_{2}\right)$ is

$$
\begin{aligned}
& \dot{V}_{2}\left(e_{6}(t), \tilde{\Theta}_{2}(t)\right) \\
= & e_{6}(t) \dot{e}_{6}(t)+\tilde{\Theta}_{2}^{T}(t) \Gamma_{2}^{-1} \dot{\tilde{\Theta}}_{2}(t) \\
= & -\alpha_{3} e_{6}^{2}(t)-m_{3} \beta_{2}\left|e_{6}(t)\right|, \quad \alpha_{3}>0, \quad m_{3} \beta_{2}>0,
\end{aligned}
$$

which indicates that the closed-loop system consisting of (73) and (74) is stable and its solutions are bounded, that is, all the variables $e_{6}(t)$ and $\tilde{\Theta}_{2}(t)$ are bounded. (76) also implies $e_{6}(t) \in L^{2} \cap L^{1}$. The boundedness of the signal $W_{2}(t)$ will also be analyzed in the following Subsection D.

\section{Overall System Stability Analysis}

In this subsection, the stability performance of the nominal and adaptive control system will be given, respectively.

Nominal control system performance. It can be seen that the nominal controllers (17) and (18) with the signals $\nu_{2}(t)$ and $\nu_{3}(t)$ designed in (51) and (55) can achieve the tracking errors $e_{2}(t)$ and $e_{6}(t)$ of the control dynamics subsystem (29)-(31) satisfying $\lim _{t \rightarrow \infty} e_{2}(t)=\lim _{t \rightarrow \infty} \dot{e}_{2}(t)=0$ exponentially and $\lim _{t \rightarrow \infty} e_{6}(t)=0$ exponentially. Further, with Lemma 2, we can have the following result:

Theorem 1: The nominal controllers (17) and (18), with the signal (51) and (55), applied to the system (7), guarantee that the corresponding closed-loop state signals $\dot{z}_{1}(t)$, $z_{1}(t)-z_{2}(t), \quad \dot{z}_{2}(t), \quad z_{2}(t)-z_{3}(t), \quad \dot{z}_{3}(t), \quad z_{3}(t)-z_{4}(t)$, $\dot{z}_{4}(t)$ are bounded, and the speed tracking errors satisfy $\lim _{t \rightarrow \infty}\left(\dot{z}_{1}(t)-v_{m}(t)\right)=0, \lim _{t \rightarrow \infty}\left(\dot{z}_{3}(t)-v_{m}(t)\right)=0$ exponentially.

\section{Proof: See Appendix A.}

Adaptive control system performance. When the system parameters are unknown, the proposed adaptive controllers (60) and (71) are used to replace the nominal controllers (17) and (18), and the stability performance is analyzed from the time derivative of the Lyapunov functions $V_{1}\left(\bar{e}_{2}, \tilde{\Theta}_{1}\right)$ and $V_{2}\left(e_{6}, \tilde{\Theta}_{2}\right)$ in (67) and (76). With Corollary 1, we have the following result:

Theorem 2: The adaptive controllers (60) and (71), with the signals (51), (55) and (65), adaptive laws (64) and (74), and $p_{12}>0$, applied to the system (7), guarantee that the corresponding closed-loop state signals $\dot{z}_{1}(t), z_{1}(t)-z_{2}(t)$, $\dot{z}_{2}(t), z_{2}(t)-z_{3}(t), \dot{z}_{3}(t), z_{3}(t)-z_{4}(t), \dot{z}_{4}(t)$ are bounded, and the speed tracking errors satisfy $\lim _{t \rightarrow \infty}\left(\dot{z}_{1}(t)-v_{m}(t)\right)=0$, $\lim _{t \rightarrow \infty}\left(\dot{z}_{3}(t)-v_{m}(t)\right)=0$.

Proof: See Appendix B.

Discussion of $p_{12}$. To guarantee the effectiveness of the proposed adaptive controller, it is required that the element $p_{12}$ in the definite positive matrix $P$ is positive. If the definite positive matrix $Q$ is chosen as a diagonal matrix, $Q=\operatorname{diag}\left\{q_{1}, q_{2}\right\}$ with $q_{1}>0$ and $q_{2}>0$, it can be calculated $p_{12}=\frac{q_{1}}{2 \alpha_{2}}>0$ from (63). Then, the following corollary can be obtained.

Corollary 2: For a diagonal definite positive matrix $Q=$ $\operatorname{diag}\left\{q_{1}, q_{2}\right\}$, the adaptive controllers (60) and (71), with the signals (51), (55) and (65), adaptive laws (64) and (74), applied to the system (7), guarantee that the corresponding closed-loop states $\dot{z}_{1}(t), z_{1}(t)-z_{2}(t), \dot{z}_{2}(t), z_{2}(t)-z_{3}(t)$, $\dot{z}_{3}(t), z_{3}(t)-z_{4}(t), \dot{z}_{4}(t)$ are bounded, and the speed tracking errors satisfy $\lim _{t \rightarrow \infty}\left(\dot{z}_{1}(t)-v_{m}(t)\right)=0, \lim _{t \rightarrow \infty}\left(\dot{z}_{3}(t)-\right.$ $\left.v_{m}(t)\right)=0$.

For the 4-car train system (7) with two control inputs acting on the 2nd and 3rd cars, the proposed nominal controller (17) and (18) and adaptive controllers (60) and (71), can guarantee partial input-to-state stability and the Lyapunov stability of the zero dynamics subsystem, desired tracking performance of the control dynamics subsystem, and the speed error having the exponential convergence under the nominal controller, and $L^{1}$ performance under the adaptive controller.

Remark 5: It should be noted that the adaptive controllers (60) and (71) are different from the corresponding nominal controllers (17) and (18), due to $\beta_{1} \operatorname{sign}\left(p_{12} e_{2}(t)+\right.$ $\left.p_{22} \dot{e}_{2}(t)\right)$ and $m_{3} \beta_{2} \operatorname{sign}\left(e_{6}\right)$. As the system parameters are unknown, the adaptive controllers (60) and (71) without the terms $\beta_{1} \operatorname{sign}\left(p_{12} e_{2}(t)+p_{22} \dot{e}_{2}(t)\right)$ and $m_{3} \beta_{2} \operatorname{sign}\left(e_{6}\right)$ can only achieve the $L^{2}$ convergence of $\xi_{1}(t)-\xi_{3}(t)$. The proposed adaptive controllers (60) and (71) can result in the $L^{1}$ convergence of $\xi_{1}(t)-\xi_{3}(t)$, which satisfies the desired convergence property of $\xi_{1}(t)-\xi_{3}(t)$, needed for ensuring the internal stability (of the zero dynamic).

Remark 6: The proposed adaptive controller design method could be extend to the case of disturbances or uncertainties. 
For the disturbances or uncertainties in the sensors which means the measurements used to construct the adaptive controller contain the noises, the filters, such as the strong tracking filter [34], or nonlinear robust filter [35], can be used to make the needed signals having the satisfied accuracy. For the modelling uncertainties, the robust adaptive technique (see e.g. [27] and [36]) can be employed.

Remark 7: So far, we have discussed adaptive controller design problems for the 4-car train model (7) with input acting on the 2nd and 3rd cars. For the high-speed trains in China, there are some other units as mentioned in Remark 1. For any unit systems, we can use the feedback linearization method to decouple the original system into a control dynamics subsystem and a zero dynamics subsystem, based on the relative degrees. For the zero dynamics subsystem, the Lyapunov and partial input-to-state stability can be obtained under certain conditions. The nominal controller and the corresponding adaptive controller can be developed to make the speeds of the control dynamics subsystem to track the desired trajectory and satisfy the needed tracking performance (exponential or $L^{1}$ ), which guarantees the stabilization condition of the zero dynamics subsystem. The proposed adaptive controller design scheme with the stabilization condition can make the partial states of the closed-loop system bounded, the speeds track the desired trajectories, and the closed-loop system satisfies the desired displacement performance, in the present of the unknown system parameters.

\section{Simulation Study}

To verify the proposed controller design method, simulation study on a real train model from [29] is presented in this section. 4 cars with two inputs acting on the 2 nd and 3 rd cars are considered, in which the general resistance and in-train forces are similar to those in Fig. 3. The parameters of the simulation are shown in Table II.

TABLE II: $\mathrm{CRH}_{2}$ train parameters

\begin{tabular}{|c|c|c|c|}
\hline Symbol & Description & Unit & Value \\
\hline$M_{1}$ & 1st car mass & ton & 42.8 \\
\hline$M_{2}$ & 2nd car mass & ton & 48 \\
\hline$M_{3}$ & 3rd car mass & ton & 46.5 \\
\hline$M_{4}$ & 4th car mass & ton & 42 \\
\hline$a_{1}, a_{4}$ & $\begin{array}{l}\text { mechanical resistance } \\
\text { coefficients }\end{array}$ & Ns/ton & 8.63 \\
\hline$a_{2}, a_{3}$ & $\begin{array}{l}\text { mechanical resistance } \\
\text { coefficients }\end{array}$ & Ns/ton & 9.03 \\
\hline$b_{1}, b_{4}$ & $\begin{array}{l}\text { mechanical resistance } \\
\text { coefficients }\end{array}$ & $\mathrm{N} \mathrm{s} /(\mathrm{m}$ ton $)$ & 0.07295 \\
\hline$b_{2}, b_{3}$ & $\begin{array}{l}\text { mechanical resistance } \\
\text { coefficients }\end{array}$ & $\mathrm{N} \mathrm{s} /(\mathrm{m}$ ton $)$ & 0.08015 \\
\hline$c$ & $\begin{array}{l}\text { aerodynamic resistance } \\
\text { coefficient }\end{array}$ & $\mathrm{N} \mathrm{s}^{2} /\left(\mathrm{m}^{2}\right.$ ton $)$ & 0.00112 \\
\hline$k_{1}, k_{3}$ & spring coefficients & $\mathrm{N} / \mathrm{m}$ & $800 \times 10^{6}$ \\
\hline$k_{2}$ & spring coefficient & $\mathrm{N} / \mathrm{m}$ & $600 \times 10^{6}$ \\
\hline$d_{1}, d_{3}$ & damper coefficients & $\mathrm{Ns} / \mathrm{m}$ & $8 \times 10^{4}$ \\
\hline$d_{2}$ & damper coefficient & $\mathrm{Ns} / \mathrm{m}$ & $6 \times 10^{4}$ \\
\hline
\end{tabular}

The initial conditions are chosen as $x(0)=$ $\left[\begin{array}{llllllll}0.5 & 0.01 & 0 & 0.01 & 0 & 0.01 & 0 & 0.01\end{array}\right]^{T}$, and the initial parameter estimates as $95 \%$ of their nominal values. The adaptive controllers proposed in (60) and (71), with the signals (51), (55) and (65) and adaptive laws (64) and (74) are used. The gains of the adaptive laws in (64) and (74) are chosen as 2 , and the parameters are chosen as $p_{12}=2$, $p_{22}=4$.

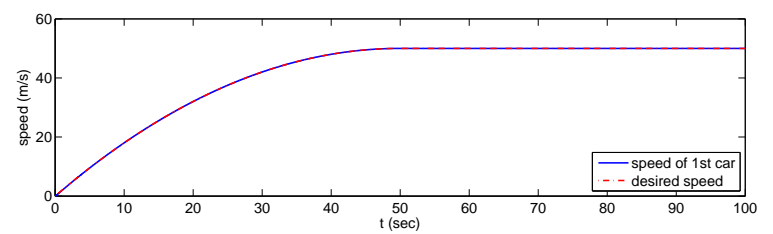

Fig. 8: Speeds of the 1st car

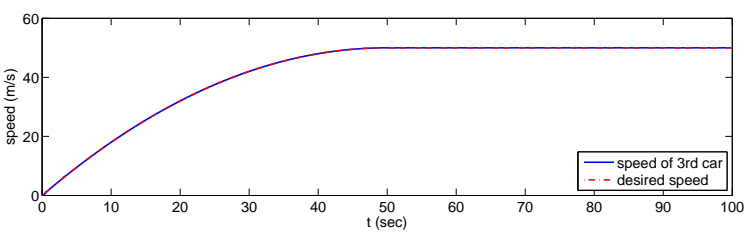

Fig. 9: Speeds of the 3rd car

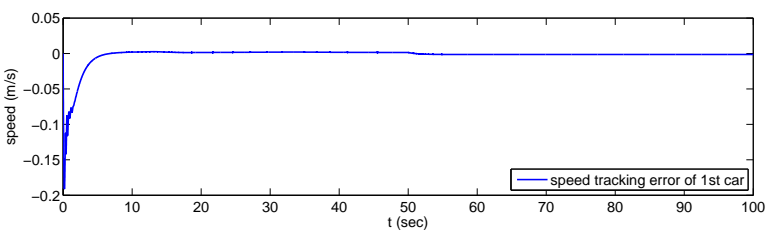

Fig. 10: Speed tracking errors of the 1st car

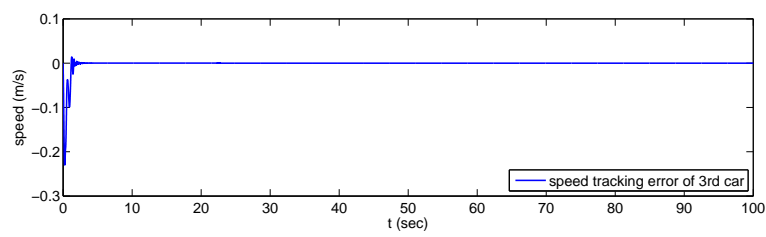

Fig. 11: Speed tracking errors of the 3rd car

Figs. 8 and 9 show the simulation results of the speeds for the 1st and 3rd cars including the plant speed (solid) and desired speed (dashed), in which the initial values of the $1 \mathrm{st}$ and 3 rd cars speeds are $0.01 \mathrm{~m} / \mathrm{s}$. Figs. 10 and 11 show the speed tracking errors for the 1st and 3rd cars. From Figs. 10 and 11 , it can be seen that the tracking errors are close to 0 . There are transit responses due to the adaptive laws and zero dynamics. Figs. 12-14 show the position of the 1st car and the relative positions between the 1 st and 2 nd cars $z_{1}(t)-z_{2}(t)$ and between the 3rd and 4th cars $z_{3}(t)-z_{4}(t)$. As the speeds of the 1 st and 3rd cars are synchronous, the error becomes a constant in steady case, which is in consistence with the real case. The simulation results show that the proposed stable adaptive control framework can achieve the close-loop stability even in the presence of unknown parameters.

It is visible from the simulation results that chattering occurs at the initial stage in figs. 10-14. This is caused by the discontinuous controllers (60) and (71) due to the sign 
function, which results in a discontinuous right hand side in the dynamical equation (7). In real implementation, the chattering can be reduced or even removed by using boundary layer method in which the discontinuous sign function is approximated by the continuous saturation function proposed in [37], [38] and [39]. Furthermore, for high-speed trains, the chattering with small amplitude usually can be accepted.

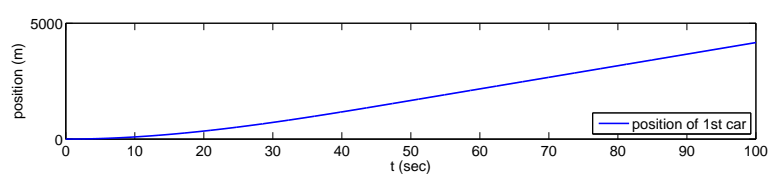

Fig. 12: Position of 1st car

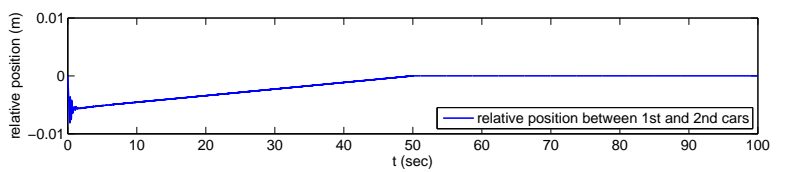

Fig. 13: Relative position between 1st and 2nd cars

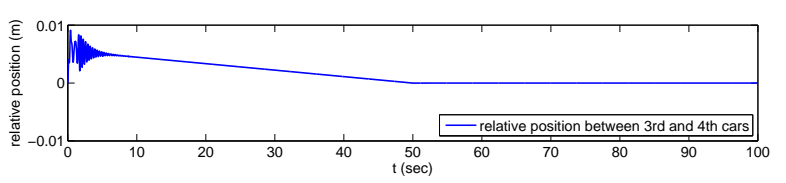

Fig. 14: Relative position between 3rd and 4th cars

\section{CONCLUSIONS}

In this paper, the adaptive tracking controller design problem has been investigated for underactuated 4-car high-speed train motion systems even if the parameters are unknown. To design the adaptive tracking controller, the nonlinear 4car train system is decoupled into a control dynamics subsystem and a zero dynamics subsystem using the feedback linearization techniques. A new and detailed stability analysis is presented to show that the zero dynamic system is the Lyapunov stable and partially input-to-state stable under the developed stabilization condition. The system configuration leads to a relative degree 1 subsystem and a relative degree 2 subsystem, for which the new adaptive controllers are proposed to ensure the needed system stabilization condition, and make the desired closed-loop system signal bounded and asymptotic speed tracking. Simulation results further confirm the obtained theoretical results.

\section{APPENDIX A}

\section{PROOF OF THEOREM 1}

When the system parameters are known, for the nominal controllers (17) and (18) with (51) and (55), it follows from (53) and (56), that $\lim _{t \rightarrow \infty} e_{2}(t)=\lim _{t \rightarrow \infty} \dot{e}_{2}(t)=0$ exponentially and $\lim _{t \rightarrow \infty} e_{6}(t)=0$ exponentially, which implies that nominal controllers $F_{2}(t)$ and $F_{3}(t)$ with $\nu_{2}(t)$ and $\nu_{3}(t)$ can make $\lim _{t \rightarrow \infty}\left(x_{2}(t)-v_{m}(t)\right)=\lim _{t \rightarrow \infty}\left(\dot{x}_{2}(t)-\right.$ $\left.\dot{v}_{m}(t)\right)=0$ exponentially, $\lim _{t \rightarrow \infty}\left(x_{6}(t)-v_{m}(t)\right)=0$ exponentially, and $\lim _{t \rightarrow \infty}\left(x_{2}(t)-x_{6}(t)\right)=0$ exponentially, i.e., $\lim _{t \rightarrow \infty}\left(\xi_{1}(t)-\xi_{3}(t)\right)=0$ exponentially.

Due to $v_{m}(t)$ and $\dot{v}_{m}(t)$ being bounded, $x_{2}(t), \dot{x}_{2}(t)$ and $x_{6}(t)$ (i.e., $\dot{z}_{1}(t), \ddot{z}_{1}(t)$ and $\dot{z}_{3}(t)$ ) are bounded, which means $\xi_{1}(t), \xi_{1}^{2}(t), \xi_{2}(t)$ and $\xi_{3}(t)$ are bounded. Considering the dynamics $\bar{G}_{1}(s)$ and $\bar{G}_{2}(s)$ in $(44), \lim _{t \rightarrow \infty}\left(\xi_{1}(t)-\xi_{3}(t)\right)=0$ exponentially results in $\eta_{2}(t), \eta_{3}(t), \eta_{4}(t), \eta_{5}(t)$ (i.e., $z_{1}(t)-$ $\left.z_{2}(t), z_{2}(t)-z_{3}(t), z_{3}(t)-z_{4}(t), \dot{z}_{4}(t)\right)$ are bounded. Recalling the inverse coordinate transformation $x_{4}(t)\left(\dot{z}_{2}(t)\right)$ in (27), and with the bounded system parameters $d_{11}, b_{1}, k_{11}, c_{1}$, and $a_{1}$, $x_{4}(t)$ is also bounded. Then, with the control signals $\nu_{2}(t)$ and $\nu_{3}(t)$ given in (51) and (55) and the structure of the nominal controllers (59) and (70), the boundedness of $F_{2}(t)$ and $F_{3}(t)$ are ensured.

\section{APPENDIX B Proof of THEOREM 2}

$L^{1}$ performance. For $(67)$, let $\epsilon(t) \triangleq p_{12} e_{2}(t)+p_{22} \dot{e}_{2}(t)$, then $e_{2}(t)=\frac{1}{p_{22} s+p_{12}}[\epsilon](t)$. According to [27], if $\frac{p_{12}}{p_{22}}>0$, $\epsilon(t) \in L^{1}$ results in $e_{2}(t) \in L^{1}$, i.e., $\xi_{1}(t)-v_{m}(t) \in L^{1}$. With $e_{6}(t)=\xi_{3}(t)-v_{m}(t) \in L^{1}$, it has $\xi_{1}(t)-\xi_{3}(t) \in L^{1}$.

Tracking error $e_{2}(t)$ performance. Moreover, $\bar{e}_{2}(t)$ is bounded, i.e., $e_{2}(t)$ and $\dot{e}_{2}(t)$ are bounded. Then, $x_{2}(t), \dot{x}_{2}(t)$ and $\hat{\Theta}_{1}(t)$ (i.e., $\hat{\theta}_{11}(t), \hat{\theta}_{12}(t), \hat{\theta}_{13}(t), \hat{\theta}_{14}(t), \hat{\theta}_{15}(t), \hat{\theta}_{16}(t)$, $\left.\hat{\theta}_{17}(t), \hat{\theta}_{18}(t), \hat{\theta}_{19}(t)\right)$ are bounded. With $e_{2}(t) \in L^{2}$ and Barbălat Lemma, $\lim _{\tilde{\sigma} \rightarrow \infty} e_{2}(t)=0$. Further, from (67), the variables $e_{6}(t)$ and $\tilde{\Theta}_{2}(t)$ are bounded. Then $x_{6}(t)$ and $\hat{\Theta}_{2}(t)$ (i.e., $\left.\hat{\theta}_{21}(t), \hat{\theta}_{22}(t), \hat{\theta}_{23}(t), \hat{\theta}_{24}(t), \hat{\theta}_{25}(t), \hat{\theta}_{26}(t), \hat{\theta}_{27}(t)\right)$ are bounded.

Zero dynamics subsystem performance. Due to $v_{m}(t)$ and $\dot{v}_{m}(t)$ being bounded and with bounded $e_{2}(t), \dot{e}_{2}(t)$ and $e_{6}(t)$, it has that $x_{2}(t), \dot{x}_{2}(t)$ and $x_{6}(t)$ are bounded, i.e., $\xi_{1}(t), \xi_{2}(t)$ and $\xi_{3}(t)$ are bounded. Then, $\xi_{1}^{2}(t)$ is bounded. Considering the dynamics $\bar{G}_{1}(s)$ and $\bar{G}_{2}(s)$ in $(44), \xi_{1}(t)-\xi_{3}(t) \in L^{1}$ and bounded $\xi_{1}(t), \xi_{1}^{2}(t), \xi_{2}(t)$ and $\xi_{3}(t)$ result in that $\eta_{2}(t)$, $\eta_{3}(t), \eta_{4}(t)$, and $\eta_{5}(t)$ are bounded, i.e., $x_{3}(t), x_{5}(t), x_{7}(t)$ and $x_{8}(t)$ are bounded.

Boundedness of $\hat{F}_{2}(t)$ and $\hat{F}_{3}(t)$. Recalling the inverse coordinate transformation $x_{4}(t)\left(\dot{z}_{2}(t)\right)$ in $(27)$, and with the bounded system parameters $d_{11}, b_{1}, k_{11}, c_{1}$ and $a_{1}, x_{4}(t)$ is also bounded. Then, according to the structure of the adaptive controllers (60) and (71), and with the bounded signals $\nu_{2}(t)$, $\nu_{3}(t)$ and $\bar{\nu}_{2}(t)$ and the bounded controller parameters $\hat{\Theta}_{1}(t)$ and $\hat{\Theta}_{2}(t)$, the boundedness of $\hat{F}_{2}(t)$ and $\hat{F}_{3}(t)$ are ensured.

Tracking error $e_{6}(t)$ performance. Further, it has that $W_{1}(t)$ and $W_{2}(t)$ are bounded. From (62) and (73), $\dot{\bar{e}}_{2}(t)$ and $\dot{e}_{6}(t)$ are bounded. With $e_{6}(t) \in L^{2} \bigcap L^{1}$ and Barbălat Lemma, $\lim _{t \rightarrow \infty} e_{6}(t)=0$.

\section{ACKNOWLEDGMENT}

Z. Mao gratefully acknowledges the support that she has received from the Department of Electrical and Computer Engineering, University of Virginia, USA. 


\section{REFERENCES}

[1] Y. B. Zhao, P. Ioannou, Positive train control with dynamic headway based on an active communication system, IEEE Trans. Intell. Transp. Syst., vol. 16, no. 6, pp. 3095-3103, Dec. 2015.

[2] Q. Song, Y. D. Song, Data-based fault-tolerant control of high-speed trains with traction/braking notch nonlinearities and actuator failures, IEEE Trans. Neural Netw., vol. 22, no. 12, pp. 2250-2261, Dec. 2011.

[3] Q. X. Yu, Z. S. Hou, J. X. Xu, D-type ILC based dynamic modeling and norm optimal ILC for high-speed trains, IEEE Trans. Control Syst. Technol., vol. 26, no. 2, pp. 652-663, March 2018.

[4] S. G. Gao, H. R. Dong, Y. Chen, B. Ning, G. R. Chen, X. X. Yang, Approximation-based robust adaptive automatic train control: an approach for actuator saturation, IEEE Trans. Intell. Transp. Syst., vol. 14, no. 4, pp. 1733-1742, Dec. 2013.

[5] C. Yang, Y. Sun, Mixed $H_{2} / H_{\infty}$ cruise controller design for high speed train, Int. J. Control, vol. 74, no. 9, pp. 905-920, June 2001

[6] Z. H. Mao, G. Tao, B. Jiang, X. G. Yan, Adaptive compensation of traction system actuator failures for high-speed trains, IEEE Trans. Intell. Transp. Syst., vol. 18, no. 11, pp. 2950-2963, Nov. 2017.

[7] L. J. Zhang, X. T. Zhuan, Optimal operation of heavy-haul trains equipped with electronically controlled pneumatic brake systems using model predictive control methodology, IEEE Trans. Control Syst. Technol., vol. 22, no. 1, pp. 13-22, Jan. 2014.

[8] Y. D. Song, Q. Song, W. C. Cai, Fault-tolerant adaptive control of highspeed trains under traction/braking failures: a virtual parameter-based approach, IEEE Trans. Intell. Transp. Syst., vol. 15, no. 2, pp. 737-748, April 2014.

[9] H. H. Ji, Z. S. Hou, R. K. Zhang, Adaptive iterative learning control for high-speed trains with unknown speed delays and input saturations, IEEE Trans. Autom. Sci. Eng., vol. 13, no. 1, pp. 260-273, Jan. 2016.

[10] S. Gao, H. Dong, B. Ning, Y. Chen, and X. Sun, Adaptive fault-tolerant automatic train operation using RBF neural networks, Neural Comput. \& Appl., vol. 26, no. 1, pp. 141-149, Jan. 2015.

[11] X. Zhuan, X. Xia, Optimal scheduling and control of heavy haul trains equipped with electronically controlled pneumatic braking systems, IEEE Trans. Control Syst. Technol., vol. 15, no. 6, pp. 1159-1166, Nov. 2007.

[12] H. Y. Tang, Q. Y. Wang, X. Y. Feng, Robust stochastic control for highspeed trains with nonlinearity, parametric uncertainty, and multiple time-varying delays, IEEE Trans. Intell. Transp. Syst., vol. 19, no. 4, pp. 1027-1037, April 2018.

[13] X. Zhuan, X. Xia, Speed regulation with measured output feedback in the control of heavy haul trains, Automatica, vol. 44, no. 1, pp. 242-247, Jan. 2008.

[14] X. Zhuan, X. Xia, Cruise control scheduling of heavy haul trains, IEEE Trans. Control Syst. Technol., vol. 14, no. 4, pp. 757-766, July 2006.

[15] S. K. Li, L. X. Yang, K. P. Li, Z. Y. Gao, Robust sampled-data cruise control scheduling of high speed train, Transp. Res. Part C: Emerg. Technol., vol. 46, pp. 274-283, Sept. 2014.

[16] H. Yang, Y. T. Fu, D. H. Wang, Multi-ANFIS model based synchronous tracking control of high-speed electric multiple unit, IEEE Trans. Fuzzy Syst., vol. 26, no. 3, pp. 1472-1484, June 2018.

[17] Y. Zhao, T. Z. Wang, H. R. Karimi, Distributed cruise control of highspeed trains, J. Frankl. Inst., vol. 354, no. 14, pp. 6044-6061, Sept. 2017.

[18] Q. Song, Y. D. Song, T. Tang, B. Ning, Computationally inexpensive tracking control of high-speed trains with traction/braking saturation, IEEE Trans. Intell. Transp. Syst., vol. 12, no. 4, pp. 1116-1125, Dec. 2011.

[19] Y. Wang, Y. D. Song, H. Gao, F. L. Lewis, Distributed fault-tolerant control of virtually and physically interconnected systems with application to high-speed trains under traction/braking failures, IEEE Trans. Intell. Transp. Syst., vol. 17, no. 2, pp. 535-545, Feb. 2016.

[20] H. Tang, Q. Wang, X. Feng, Robust stochastic control for high-speed trains with nonlinearity, parametric uncertainty, and multiple timevarying delays, IEEE Trans. Intell. Transp. Syst., vol. 19, no. 4, pp. 1027-1037, April 2018.

[21] A. Isidori, Nonlinear Control Systems 3rd ed. Berlin, German: Springer Verlag, 1995.

[22] H. K. Khalil, Nonlinear Systems, 3rd ed. Upper Saddle River, NJ, USA: Prentice Hall, 2001.

[23] L. Le-Tien, A. Albu-Schäffer, Robust adaptive tracking control based on state feedback controller with integrator terms for elastic joint robots with uncertain parameters, IEEE Trans. Control Syst. Technol., vol. 26, no. 6, pp. 2259-2267, Nov. 2018.
[24] J. P. Cai, C. Y. Wen, H. Y. Su, Z. T. Liu, Robust adaptive failure compensation of hysteretic actuators for a class of uncertain nonlinear systems, IEEE Trans. Autom. Control, vol. 58, no. 9, pp. 2388-2394, Sept. 2013.

[25] W. He, T. T. Meng, Adaptive control of a flexible string system with input hysteresis, IEEE Trans. Control Syst. Technol., vol. 26, no. 2, pp. 693-700, March 2018.

[26] Z. J. Li, Y. P. Yang, J. X. Li, Adaptive motion/force control of mobile under-actuated manipulators with dynamics uncertainties by dynamic coupling and output feedback, IEEE Trans. Control Syst. Technol., vol. 18, no. 5, pp. 1068-1079, Sept. 2010.

[27] G. Tao, Adaptive Control Design and Analysis. New York, NY, USA: Wiley, 2003.

[28] V. K. Garg, Dynamics of railway vehicle systems. Ontario, Academic Press Canada, 1984

[29] S. G. Zhang, Fundamental Application Theory and Engineering Technology for Railway High-Speed Trains, Beijing, China: Science Press, 2007.

[30] M. Chou, X. Xia, C. Kayser, Modelling and model validation of heavyhaul trains equipped with electronically controlled pneumatic brake systems, Control Eng. Pract., vol. 15, no. 4, pp. 501-509, April 2007.

[31] B. P. Rochard, F. Schmid, A review of methods to measure and calculate train resistances, Proc IMechE, Part F: J Rail Rapid Transit, vol. 214 , no. 4, pp. 185-199, July 2000 .

[32] E. D. Sontag, Input to State Stability: Basic Concepts and Results, Nonlinear and Optimal Control Theory, Springer, 2004, 163-220.

[33] Y. J. Zhang, G. Tao, M. Chen, Relative degrees and adaptive feedback linearization control of T-S fuzzy systems, IEEE Trans. Fuzzy Syst., vol. 23, no. 6, pp. 2215-2230, Dec. 2015.

[34] X. He, Z. D. Wang, X. F. Wang, D. H. Zhou, Networked strong tracking filtering with multiple packet dropouts: algorithms and applications, IEEE Trans. Ind. Electron., vol. 61, no. 3, pp. 1454-1463, March 2014.

[35] X. He, Z. D. Wang, D. H. Zhou, Robust $\mathrm{H}_{\infty}$ filtering for time-delay systems with probabilistic sensor faults, IEEE Signal Process. Lett., vol. 16 , no. 5 , pp. 442-445, May 2009.

[36] P. A. Ioannou, J. Sun, Robust Adaptive Control, Englewood Cliffs, NJ, USA: Prentice-Hall, 1996.

[37] J. A. Burton, A. S. I. Zinober, Continuous approximation of variable structure control, Int. J. Syst. Sci., vol. 17, no. 6, pp. 875-885, June 1986.

[38] F. Esfandiari and H. K. Khalil, Stability analysis of a continuous implementation of variable structure control, IEEE Trans. Autom. Control, vol. 36, no. 5, pp. 616-620, May 1991.

[39] C. Edwards, S. Spurgeon, Sliding Mode Control: Theory \& Applications. London, U.K.: Taylor \& Francis, 1998.

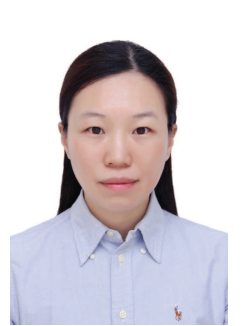

Zehui Mao (M'10) received her Ph.D. degree in Control Theory and Control Engineering from Nanjing University of Aeronautics and Astronautics, Nanjing, China, in 2009. She is currently a professor at the College of Automation Engineering in Nanjing University of Aeronautics and Astronautics, China. She was a visiting scholar in University of Virginia. She worked in the areas of fault diagnosis, with particular interests in nonlinear control systems, sampled-data systems and networked control systems. Her current research interests include fault diagnosis and fault-tolerant control of systems with disturbance and incipient faults, and high speed train and spacecraft flight control applications. 


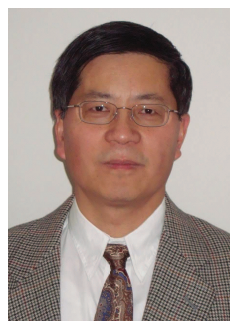

Gang Tao (S'84-M'89-SM'96-F'07) received the B.S. degree from University of Science and Technology of China in 1982, and the M.S. and Ph.D. degrees from University of Southern California, USA, during 1984-1989. He is currently a Professor at University of Virginia, USA. He worked in the areas of adaptive control, and he has authored or coauthored 8 books, and more than 450 technical papers and book chapters. His current research interests include adaptive control of systems with uncertain actuator failures and nonlinearities, with structural damage and sensor uncertainties and failures, adaptive approximation-based control, and resilient aircraft and spacecraft flight control applications. He has served as an Associate Editor of Automatica, International Journal of Adaptive Control and Signal Processing, and IEEE Transactions on Automatic Control, a Guest Editor of Journal of Systems Engineering and Electronics, and an Editorial Board Member of the International Journal of Control, Automation and Systems.

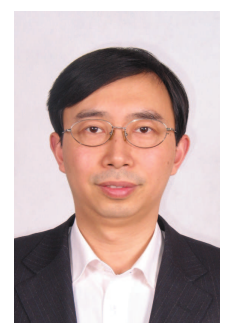

Bin Jiang (M'03-SM'05-F'20) received the Ph.D. degree in automatic control from Northeastern University, Shenyang, China, in 1995. He had ever been a Post-Doctoral Fellow, a Research Fellow, an Invited Professor, and a Visiting Professor in Singapore, France, USA, and Canada, respectively. He is currently Chair Professor of Cheung Kong Scholar Program with the Ministry of Education and the Vice President of Nanjing University of Aeronautics and Astronautics, Nanjing, China. He has authored eight books and over 200 referred international journal papers and conference papers. His current research interests include intelligent fault diagnosis and fault tolerant control and their applications to helicopters, satellites, and high-speed trains. Dr. Jiang was a recipient of the Second Class Prize of National Natural Science Award of China in 2018. Dr. Jiang is a Fellow of Chinese Association of Automation (CAA). He currently serves as an Associate Editor or an Editorial Board Member for a number of journals, such as the IEEE Trans. On Cybernetics, Journal of the Franklin Institute, Neurocomputing at, $\mathrm{He}$ is a Chair of Control Systems Chapter in IEEE Nanjing Section, a member of IFAC Technical Committee on Fault Detection, Supervision, and Safety of Technical Processes.

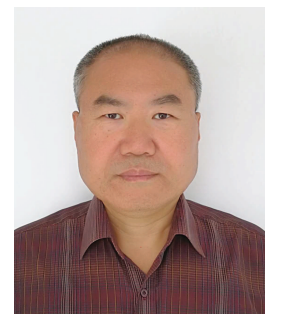

Xing-Gang Yan received B.Sc. degree from Shanxi Normal University, in 1985, M.Sc. degree from Qufu Normal University in 1991, and Ph.D. degree in Engineering from Northeastern University, China in 1997. Currently, he is a Senior Lecturer at the University of Kent, UK. He was a Lecturer at Qingdao University, China from 1991 to 1994. He worked as a Research Fellow/Associate in the University of Hong Kong, China, Nanyang Technological University, Singapore and the University of Leicester,

UK. He is the Editor-in-Chief of the International Journal of Engineering Research and Science and Technology. His research interests include sliding mode control, decentralised control, fault detection and isolation, control and observation of nonlinear systems and time delay systems with applications. 